\title{
Les transformations de contact entre les éléments fondamentals de l'espace.
}

\author{
(Par E.-O. Lovetr, à Princeton, New Jersey, États-Unis d'Amérique.)
}

Le rôle capitale des notions de transformation et groupe dans la géométrie moderne mis en evidence par l'ourrage de Sophus Lie et les Mémoires de MM. Branchi, Kiein, Ponncaré, et autres, est l'inspiration de ce travail qui s'occupe avec les transformations de contact entre les éléments les plus essentiels de l'espace, savoir multiplicités singulières, sphères, surfaces développables ou applicables, lignes asymptotiques et lignes de courbure, et qui, en ce qui concerne la géométrie de l'espace à plusieurs dimensions, se base sur un Mémoire recent, Journal de $M$. Jordan, 1901, où on trouve une application de la théorie de groupes finis à la construction des éléments de la géométrie euclidienne ordinaire et différentielle de l'espace à $n$ dimensions, et une application de la théorie de groupes infinis à la théorie de la déformation de variétés euclidiennes et non-euclidiennes.

DÉFINITIONS ET MÉTHODES DE DÉTERMINATION DES TRANSFORMATIONS DE CONTACT DE L'ESPACE $\mathbf{a} n+1$ DIMENSIONS.

Un élément de surface est l'ensemble d'un point et d'un plan passant par ce point; il a pour coordonnées les coordonnées cartésiennes $\left(z, x_{1}\right.$, $\left.x_{2}, \ldots, x_{n}\right)$ du point et les coefficients de direction $p_{1}, p_{2}, \ldots, p_{n}$ du plan

$$
Z-z=\sum_{i}^{n} p_{i}\left(X_{i}-x_{i}\right)
$$


Une multiplicité d'éléments est définie par un système d'équations, entre les coordonnées $z, x_{1}, x_{2}, \ldots, x_{n}, p_{1}, p_{2}, \ldots, p_{n}$ de l'un quelconque de ses éléments, satisfaisant à l'équation de PFAFF

$$
d z-p_{1} d_{1} x-\cdots-p_{n} d x_{n}=0 \text {. }
$$

Si ce système se compose de $n+1$ équations distinctes, il définit une multiplicité $M_{n}$ d'éléments.

LiE dit encore que l'équation de Pfaff (2) exprime que l'élément ( $z$, $\left.x_{1}, x_{2}, \ldots, x_{n}, p_{1}, p_{2}, \ldots, p_{n}\right)$ et l'élément infiniment roisin $(z+d z$, $\left.x_{1}+d x_{1}, \ldots, x_{n}+d x_{n}\right)$ sont unis. Une multiplicité est alors une famille d'éléments dans laquelle chaque élément est uni à tout élément infiniment voisin de la famille.

On dit que la transformation

$$
\begin{aligned}
\boldsymbol{z}^{\prime} & =Z\left(z, x_{1}, x_{2}, \ldots, x_{n}, p_{1}, p_{2}, \ldots, p_{n}\right) \\
x^{\prime}{ }_{i} & =X_{i}(z, x, p), \\
p^{\prime}{ }_{i} & =P_{i}(z, x, p),
\end{aligned} \quad i=1,2, \ldots, n,
$$

est une transformation de contact de l'espace $a n+1$ dimensions $\left(z, x_{1}\right.$, $\left.x_{2}, \ldots, x_{n}\right)$ si elle laisse invariante l'équation de Pfafr (2).

Il résulte immédiatement de là que ces transformations forment un groupe. et sont deux à deux inverses l'une de l'autre.

La définition des transformations de contact est susceptible de prendre une forme géométrique si l'on employe les notions de multiplicités d'éléments et d'éléments unis. On peut définir une transformation de contact comme une transformation d'éléments qui change toujours des éléments unis en éléments unis, c'est-à-dire qui transforme des multiplicités $M_{n}$ en multiplicités $M_{n}$, et Liw a montré que les transformations de contact sont les seules transformations en $z, \alpha, p$ qui changent tout système de $n+1$ équations, satisfaisant à l'équation de Prafe (2), en un système jouissant la même propriété.

On peut faire la détermination des formes explicites des équations défnissantes d'une transformation de contact des manières suivantes:

1. ${ }^{\circ} \mathrm{Au}$ moyen de l'opération d'intégration en employant le théorème de Lre, Darboux et Maxer. Pour. que les équations (3) définissent une transformation. de contact il faut et il suffit que l'on ait une identité de la forme

$$
d z-p_{1} d x_{1}-\cdots-p_{n} d x_{n}=\frac{1}{i}\left(d Z-P_{4} d X_{1}-\cdots-P_{n} d X_{n}\right) .
$$


Iire, Darboux et Mayer en déduisent qu'il faut et il suffit que les fonctions $Z, X_{1}, \ldots, X_{n}, P_{1}, \ldots, P_{n}$ soient liées par des relations identiques de la forme

$$
\left.\begin{array}{l}
\left(Z, X_{i}\right)=\left(X_{i}, X_{k}\right)=\left(P_{i}, X_{k}\right)=0, \quad(i=k=k) \\
\left(P_{i}, X_{i}\right)=\rho, \quad\left(P_{i}, Z\right)=\rho P_{i} .
\end{array}\right\}
$$

$2 .^{\circ} \mathrm{Au}$ moyen des opérations de différentiation et élimination en employant le théorème de LuE qui dit en effet qu'on obtient toutes les transformations de contact en $z, x_{1}, \ldots, x_{n}, p_{1}, \ldots, p_{n}$ en éliminant les indéterminées $\lambda$. entre des équations de la forme

$$
\begin{gathered}
\Omega_{i}\left(z, x_{1}, \ldots, x_{n}, z^{\prime}, x_{1}^{\prime}, \ldots, x_{n}^{\prime}\right), \quad \Omega_{2}\left(z, x, z^{\prime}, x^{\prime}\right)=0, \ldots, \\
\Omega_{q}\left(z, x, z^{\prime}, x^{\prime}\right)=0 \\
p_{i}=-\frac{\lambda_{1} \frac{\partial \Omega_{1}}{\partial x_{i}}+\cdots+\lambda_{q} \frac{\partial \Omega_{q}}{\partial x_{i}}}{\lambda_{1} \frac{\partial \Omega_{1}}{\partial z}+\cdots+\lambda_{q} \frac{\partial \Omega_{q}}{\partial z^{\prime}}}, \\
p_{i}^{\prime}=-\frac{\lambda_{1} \frac{\partial \Omega_{1}}{\partial x_{i}}+\cdots+\lambda_{q} \frac{\partial \Omega_{q}}{\partial x_{i}}}{\lambda_{1} \frac{\partial \Omega_{1}}{\partial z^{\prime}}+\cdots+\lambda_{q} \frac{\partial \Omega_{q}}{\partial z^{\prime}}}, \quad i=1,2, \ldots, n .
\end{gathered}
$$

Pour les définitions et théorèmes précedents on peut consulter

Goursat, Lecgons sur les équations aux dérivées partielles du premier ordre.

LIE-Enaes, Theorie der 'Transformationsgruppen, tome II.

LiE-Scheffers, Geometrie der Beriuhrungstransformationen, tome I.

LES TRANSFORMATIONS DE CONTACT ENTRE LES MUITIPLICITÉS SINGULILRES.

Entre les multiplicités des éléments de surface ils existent quelques unes qui ont la propriété que tous ses éléments sont deux à deux des éléments unis. Il est peut-être permissible de nommer telles multiplicités des éléments multiplicités singulières. On trouve trois types de ces multiplicités singulières: la famille de tous les éléments de surface d'un point, l'agrégat de tous 
les éléments de surface d'une ligne droite, et l'ensemble de tous les éléments de surface d'un plan. Le problème de déterminer toutes les transformations de contact qui changent les multiplicités singalières en multiplicités singulières demande qu'on détermine les catégories snivantes de transformations de contact: $1 .^{\circ}$ les transformations des points en points; $2 .^{\circ}$ les transformations qui changent les points en points et les plans en plans; $3 .^{\circ}$ les transformations qui changent les points en plans et les plans en points; $4 .^{\circ}$ les transformations des droites en droites; $5 .^{\circ}$ les transformations entre les plans.

On sait bien que la première catégorie se compose du groupe infini de toutes les transformations ponctuelles prolongées; ils sont définies par $n+1$ équations directrices entre les coordonnées ponctuelles des espaces correspondants. La deuxième catégorie consiste en le groupe fini des transformations projectives; ces transformations sont déterminées au moyen de $n+1$ équations directrices bilinéaires; on établira ces résultats connus dans la suite s'en servant d'une méthode déjà employée dans le plan (LiE-Schefrers, Vorlesungen über continuierliche Gruppen). La troisième catégorie se construit de toutes les transformations dualistiques; ces transformations-ci connues se déterminent d'une équation directrice bilinéaire. La quatrième catégorie se compose des transformations des deuxième et troisième catégories, savoir des transformations projectives et dualistiques; ce résultat connue se révèle immédiatement de notre définition de multiplicité singulière. On dérive la forme et quelques propriétés de la cinquième catégorie dans les pages suivantes.

Les TRANSFORMATIONS DE CONTACT

QUI ChaNgent LES POINTS EN POINTS ET LES PLANS EN PLANS.

Employons nous, pour la resolution de ce problème la méthode des transformations infinitésimales. Soit

$$
U f \equiv \zeta \frac{\partial f}{\partial z}+\sum_{i=1}^{n} \xi_{i} \frac{\partial f}{\partial x_{i}}
$$

la transformation infinitésimale, où les fonctions $\xi_{i}$ et $\zeta$ sont des fonctions de $x_{1}, x_{2}, \ldots, x_{n}, z$ qui doivent être déterminées de façon telle que la transformation soit une plan-en-plan transformation; la condition nécessaire et suffisante pour cette détermination est que les équations aux dérivées ar- 
tielles

$$
p_{i j} \equiv \frac{\partial^{2} z}{\partial x_{i} \partial x_{j}}=0, \quad i, j=1,2, \ldots, n,
$$

ne changent pas sous la transformation.

Les variations des $x_{j}$ sous la transformation infinitésimale ponctuelle $U f$ sont

$$
\delta x_{j}=\xi_{j}\left(x_{1}, x_{2}, \ldots, x_{n}, z\right) \delta t, \quad j=-1,2, \ldots, n
$$

où $\delta t$ est une infinitésimale arbitraire.

Pour déterminer les variations de $p_{i}$ et $p_{i j}$ il est nécessaire à construire Je second prolongement

$$
U^{\prime \prime} f=U f+\sum_{1}^{n} \pi_{i} \frac{\partial f}{\partial p_{i}}+\mathbf{\Sigma}_{i}^{n} \mathbf{\Sigma}_{j} \pi_{i j} \frac{\partial f}{\partial p_{i j}}
$$

de la transformation $U f$. On fait cette construction de la manière suivante: - En variant l'identité

$$
d z-\sum_{i}^{n} p_{i} d x_{i}=0
$$

on a

$$
d \delta z=\sum_{1}^{n}\left(d x_{i} \delta p_{i}+p_{i} d \delta x_{i}\right)
$$

en substituant les valeurs (3) des variations $\delta x_{i}$ et en observant que l'équation (6) doit exister identiquement pour toutes les valeurs des $d x_{i}$ on trouve les $n$ équations suivantes pour la détermination des variations $\delta p_{i}$ :

$$
\zeta_{x_{i}}+p_{i} \zeta_{z}=o p_{i}+\sum_{1}^{n} j p_{j}\left(\xi_{j x_{i}}+p_{i} \xi_{j_{z}}\right), \quad i=1,2, \ldots, n,
$$

ou

$$
\pi_{i} \equiv \delta p_{i}=\zeta_{x_{i}}+p_{i} \zeta_{z}-\sum_{i}^{n} j p_{j}\left(\xi_{j x_{i}}+p_{i} \xi_{j z}\right) .
$$

De la même façon on varie les identités

$$
d p_{j}=\sum_{i}^{n} p_{i j} d x_{i}
$$

et trouve les valeurs suivantes pour les variations des $p_{i j}$

$$
\pi_{i j} \equiv \partial p_{i j}=\tau_{i x_{j}}+p_{j} \pi_{i z}+\sum_{i}^{n} p_{i j} \pi_{i_{j}}-\sum_{\mathbf{I}}^{n} p_{i_{j}}\left(\xi_{j x_{j}}+p_{i} \xi_{j_{z}}\right) .
$$

Pour que la famille de tous les $\infty^{n+1}$ plans-de l'espace soit invariante 
sous la transformation $U f$ il faut que les équations

$$
\pi_{i j}=0, \quad i, j=1,2, \ldots, n,
$$

aient lieu en conséquence des équations (2); donc on a les équations de condition suivantes :

$$
\begin{gathered}
\zeta_{x x_{0} \infty_{j}}=0, \quad i, j=1,2, \ldots, n ; \\
\xi_{i_{z z}}=0, \quad \xi_{i_{x_{j} x_{j}}}=0, \quad \xi_{i_{x_{j} z}}=0, \quad i, j=1,2, \ldots, n, \quad j=\mid=i ; \\
2 \zeta_{z x_{i}}-\xi_{i_{x_{i} x_{j}}}=0, \quad \zeta_{z z}-2 \xi_{i_{x_{i z}}}=0, \quad i=1,2, \ldots, n ; \\
\zeta_{z x_{i}}-\xi_{j_{x_{i} x_{j}}}=0, \quad \zeta_{z z}-\xi_{i_{x_{i} z}}-\xi_{j_{x_{j} z}}=0, \quad i, j=1,2, \ldots, n, \quad i=\mid=j .
\end{gathered}
$$

Les équations (12) demandent que la fonction $\xi_{i}$ soit linéaire dans toutes les $x_{j}$ avec exception de $x_{i}$, contenant aucune produit de la forme $x_{j} z$, où $j=i$. D'ailleurs les mêmes équations disent que la fonction $\varphi$ est une fonction linéaire des quantités $x_{1}, x_{2}, \ldots, x_{n}$ sans termes de la forme $x_{i} x_{j}$. Donc on a

$$
\left.\begin{array}{ll}
\xi_{i}=\sum_{1}^{n} \varphi_{j}\left(x_{i}\right) x_{j}+\psi\left(x_{i}\right) z+\chi\left(x_{i}\right), & j=i=i \\
\zeta=\sum_{1}^{n} \rho_{j}(z) x_{j}+\sigma(z), & j=1,2, \ldots, i, \ldots, n .
\end{array}\right\}
$$

On voit aussi que l'équation

$$
\rho_{j}=\alpha_{j}+\beta_{j}
$$

doit avoir lieu en conséquence de l'équation

$$
\zeta_{x_{j} z z}=0
$$

et encore les équations (13) montrent que

$$
\begin{array}{ll}
\varphi_{j}\left(x_{i}\right) \equiv \alpha_{i} x_{i}+\gamma_{i}, & \psi\left(x_{i}\right) \equiv A x_{i}+B, \\
\chi\left(x_{i}\right) \equiv \alpha_{i} x_{i}^{2}+\delta_{i} x_{i}+\sigma_{i}, & \sigma(z) \equiv A z^{2}+C z+D ;
\end{array}
$$

donc en changeant commodément les constantes et en écrivant

on a enfin

$$
V \equiv \sum_{i}^{n} a_{i} x_{i}+b_{i}
$$

$$
\left.\begin{array}{l}
\xi_{i}=\sum_{1}^{n} \alpha_{i j} x_{j}+\beta_{i} z+x_{i} V+\gamma_{i}, \\
\zeta=\sum_{1}^{n} \lambda_{j} x_{j}+\mu z+z V+\nu .
\end{array}\right\}
$$


D'après un théorème bien connu de Lre les transformations finies engendrées par la transformation ponctuelle infinitesimale (1) sont données par l'intégration du système

$$
\left.\begin{array}{c}
\frac{d z^{\prime}}{\zeta\left(x_{1}^{\prime}, x_{2}^{\prime}, \ldots, x_{n,}^{\prime} z^{\prime}\right)}=\frac{d x_{1}^{\prime}}{\xi_{1}\left(x_{1}^{\prime}, x_{2}^{\prime}, \ldots, x_{n}^{\prime}, z^{\prime}\right)}=\cdots \\
\cdots=\frac{d z_{n}^{\prime}}{\xi_{n}\left(x_{1}^{\prime}, x_{1}^{\prime}, \ldots, x_{n}^{\prime}, z^{\prime}\right)}=d t,
\end{array}\right\}
$$

avec les conditions initiales

$$
x_{1}^{\prime}=x_{1}, \quad x_{2}^{\prime}=x_{2}, \ldots, \quad x_{n}^{\prime}=x_{n}, \quad z^{\prime}=z, \quad t=0 ;
$$

ce qui donne

$$
x_{1}^{\prime}=\frac{L_{1}}{N}, \quad x_{2}^{\prime}=\frac{L_{2}}{N}, \ldots, \quad x_{n}^{\prime}=\frac{L_{n}}{N}, \quad z^{\prime}=\frac{M}{N},
$$

où les fonctions $L_{1}, L_{2}, \ldots, L_{n}, M, N$, sont de la forme

$$
\sum_{1}^{n} l_{j} x_{j}+m z+n
$$

les $l_{j}, m, n$ étant des constantes quelconques.

Les transformations de contact qui changent les plans en plans;

LES TRANSFORMATIONS ENTRE SURFACES DÉVELOPPABLES.

Il est clair qu'on obtient la transformation de contact la plus générale entre tous les plans de l'espace à $n+1$ dimensions en formant tous les produits

$$
D P D
$$

où $D$ est la transformation dualistique générale et $P$ est une transformation ponctuelle tout-à-fait arbitraire.

Pour écrire la forme analytique des transformations on peut prendre pour la transformation dualistique $D$ sans perte de généralité la transformation généralisée de LEgENDre

$$
X_{i}=p_{i}, \quad Z=\zeta=\sum_{i}^{n} p_{i} x_{i}-z, \quad P_{i}=x_{i}, \quad i=1,2, \ldots, n .
$$


De la transformation ponctuelle arbitraire $P$

$$
X_{i}^{\prime}=\varphi_{i}\left(x_{1}^{\prime}, x_{2}^{\prime}, \ldots, x_{n}^{\prime}, z^{\prime}\right), \quad Z^{\prime}=\varphi_{0}\left(x_{1}^{\prime}, x_{2}^{\prime}, \ldots, x_{n}^{\prime}, z^{\prime}\right)
$$

on deduit

done

$$
d X_{i}^{\prime}=\sum_{1}^{n}{ }_{j}\left(\varphi_{i z^{\prime}}, p_{j}^{\prime}+\varphi_{i x_{j}^{\prime}}\right) d x_{j}^{\prime}, \quad d Z^{\prime}=\sum_{1}^{n}\left(\varphi_{0 z^{\prime}} p_{j}^{\prime}+\varphi_{0 x^{\prime}}\right) d x_{j}^{\prime} ;
$$

$$
\sum_{i}^{n} P_{i}^{\prime} d X^{\prime}{ }_{i}=\sum_{i}^{n}\left(\varphi_{0 z^{\prime}} p^{\prime}{ }_{i}+\rho \varphi_{x_{i}^{\prime}}\right) d x^{\prime}{ }_{i}=\sum_{i}^{n}{ }_{i} P^{\prime}{ }_{i} \sum_{i}^{n}\left(\varphi_{i z^{\prime}} p_{j}^{\prime}+\varphi_{i z_{j}^{\prime}}\right) d x_{j}^{\prime},
$$

et encore

$$
\sum_{1}^{n} P_{i}^{\prime}\left(\varphi_{i z^{\prime}} p_{j}^{\prime}+\varphi_{i x_{j^{\prime}}}\right)=\varphi_{\theta_{z^{\prime}}} p_{j}^{\prime}+\varphi_{0_{x_{j}^{\prime}}}, \quad j=1,2, \ldots, n .
$$
donne

La résolution de ce système des équations linéaires pour les quantités $P^{\prime}{ }_{i}$

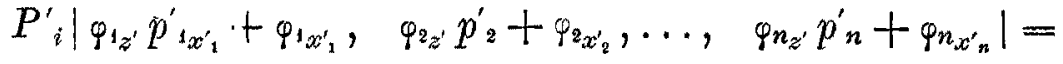

$$
\begin{aligned}
& \mid \varphi_{z_{z^{\prime}}} p_{1}^{\prime}+\varphi_{x_{1}^{\prime}}, \quad \varphi_{2 z^{\prime}} p_{z}^{\prime}+\varphi_{z_{x_{2}^{\prime}}}, \ldots, \varphi_{i-1 z^{\prime}} p_{i-1}^{\prime}+\varphi_{i-4 x_{i-1}^{\prime}}, \\
& \left.\varphi_{0_{z^{\prime}}} p^{\prime}{ }_{i}+\varphi_{x_{x^{\prime}}}, \quad \varphi_{i+z_{z^{\prime}}} p_{i+3}^{\prime}+\varphi_{i+3}{ }_{x_{i+1}^{\prime}}, \ldots, \quad \varphi_{n_{z^{\prime}}} p_{n}^{\prime}+\varphi_{n_{x^{\prime}}} \mid .\right)
\end{aligned}
$$

Enfin en appliquant la transformation de LEGeNDRE aux variables originals et aux variables transformés, et en écrivant

$$
M_{1} \equiv\left(H_{1}, H_{2}, \ldots, H_{n}\right)_{1}, \ldots, \quad M_{n+1} \equiv\left(H_{1}, H_{2}, \ldots, H_{n}\right)_{n+1}
$$

pour les mineurs de $m_{1}, m_{2}, \ldots, m_{n+1}$ du déterminant

$$
\left|\frac{\partial H_{1}}{\partial \zeta}, \frac{\partial H_{2}}{\partial p_{1}}, \frac{\partial H_{3}}{\partial p_{2}}, \ldots, \frac{\partial H_{n}^{*}}{\partial p_{n-1}}, m_{n+1}\right|
$$

nous trouvons pour les équations explicites des plan-en-plan transformations de contact

$$
\begin{gathered}
X_{i} \stackrel{\sum}{0} j_{j}^{n} x_{j}\left(\varphi_{1}, \varphi_{2}, \ldots, \varphi_{n}\right)_{j+1}= \\
=\sum_{0}^{n} x_{j}\left(\varphi_{i+1}, \varphi_{i+2}, \ldots, \varphi_{n}, \varphi_{0}, \varphi_{1}, \ldots, \varphi_{i-1}\right)_{j+1} \\
x_{0}=1 ; i=1,2, \ldots, n ; \\
Z=\sum_{i}^{n} X_{i} \varphi_{i}\left(\zeta, p_{1}, p_{2}, \ldots, p_{n}\right)-\varphi_{0}\left(\zeta, p_{1}, p_{2}, \ldots, p_{n}\right) \\
P_{i}=\varphi_{i}\left(\zeta, p_{1}, p_{2}, \ldots, p_{n}\right), \zeta=\sum_{i}^{n} p_{i} x_{i}^{\prime}-z
\end{gathered}
$$

où les fonctions $\varphi_{0}, \varphi_{1}, \ldots, \varphi_{n}$ sont des fonctions arbitraires. 
On observe en passant que les fonctions $P_{i}$ satisfont aux équations aux dérivées partielles

$$
P_{i_{x_{j}}}+p_{j} P_{i_{z}} \equiv P_{(i j)}=0, \quad i, j=1,2, \ldots, n ;
$$

on peut vérifier ce fait soit directement par différentiation ou par la considération suivante.

Les équations aux dérivées partielles du second ordre

$$
p_{i j} \equiv \frac{\partial^{2} z}{\partial x_{i} \partial x_{j}}=0, \quad i, j=1,2, \ldots, n
$$

sont des invariants sous les transformations de contact entre les plans.

En posant

$$
F^{(i)}=F_{x_{i}}+p_{i} F_{z}+\sum_{i}^{n} p_{i j} F_{p_{j}}
$$

en vertu des identités

$$
d Z=\sum_{\mathbf{I}}^{n} P_{i} d X_{i}, \quad d P_{i}=\sum_{\mathbf{1}}^{n} P_{i j} d X_{j}
$$

on a

$$
P_{j}^{(i)}=\Sigma k P_{j k} x_{k}^{(i)}, \quad i, j=1,2, \ldots, n
$$

la résolution de ce système des équations linéaires pour $P_{j k}$ donne

$$
P_{i j}=\frac{\Phi\left(X_{1}, X_{2}, \ldots, X_{i-1}, P_{j}, X_{i+1}, \ldots, X_{n}\right)}{\Phi\left(X_{i}, X_{2}, \ldots, X_{n}\right)}=\frac{\Phi\left(X_{i}, X_{2}, \ldots, X_{j-1} P_{i}, X_{j+1}, \ldots, X_{n}\right)}{\Phi\left(X_{i}, X_{2}, \ldots, X_{n}\right)},
$$

où

$$
\Phi\left(T_{1}, T_{2}, \ldots, T_{n}\right) \equiv\left|T_{1}^{(1)}, T_{2}^{(2)}, \ldots, T_{n}^{(n)}\right|
$$

Donc pour l'invariance de la famille de tous les plans de l'espace il faut que

$$
\left.\begin{array}{l}
\Phi\left(X_{1}, X_{2}, \ldots, X_{i-1}, X_{i}, X_{i+1}, \ldots, X_{n}\right)=0, \\
\Phi\left(X_{1}, X_{2}, \ldots, X_{i-1}, P_{j}, X_{i+1}, \ldots, X_{n}\right)=0, \quad i, j=1,2, \ldots, n \\
\Phi\left(X_{1}, X_{2}, \ldots, X_{j+1}, P_{i}, X_{j+1}, \ldots, X_{n}\right)=0,
\end{array}\right\}
$$

soient vraies pour toutes les valeurs de $p_{1}, p_{2}, \ldots, p_{n}$; donc on a

$$
\left.\begin{array}{c}
\Delta \equiv\left|X_{(11)}, X_{(22)}, \ldots, X_{(n n)}\right|=\mid=0, \\
\left|X_{(11)}, X_{(22)}, \ldots, X_{(i-1 i-1)}, P_{(j j)}, X_{(i+1 i+1)}, \ldots, X_{(n n)}\right|=0, \\
\left|X_{(11)}, X_{(22)}, \ldots, X_{(j-1 j-1)}, P_{(i i)}, X_{(j+1 j+1)}, \ldots, X_{(n n)}\right|=0 ;
\end{array}\right\}
$$


en observant que les équations (20) sont des $n$ systèmes des équations linéaires dont les déterminants sont le déterminant réciproque de $\Delta$, on a

$$
P_{(i 1)}=0, \quad P_{(i \varepsilon)}=0, \ldots, \quad P_{(i n)}=0, \quad i=1,2, \ldots, n \text {. }
$$

D'ailleurs l'application de la méthode de M. MAYER à ces systèmes complets donne

$$
P_{i}=\varphi_{i}\left(\zeta, p_{1}, p_{2}, \ldots, p_{n}\right), \quad i=1,2, \ldots, n,
$$

où les fonctions $\varphi_{i}$ sont des fonctions quelconques.

Enfin on a en même temps la résolution d'un problème plus générale. En effet l'équation

$$
\left|p_{11}, p_{22}, \ldots, p_{n n}\right|=0
$$

doit être invariante sous les transformations entre les surfaces développables de l'espace à $n+1$ dimensions. Il est facile de vérifier que les conditions nécessaires et suffisantes sont exprimées par les équations (21); done la famille de transformations de contact qui transforment une surface développable quelconque en une surface développable est identique à la famille de toutes les transformations de contact entre les plans.

Les transformations de CONTACT ENTRE LES LIGNES DROITES ET LES SPHìres.

Une des plus belles découvertes de la Géométrie moderne est la transformation de contact connue sous le nom de transformation de LiE qui établit une liaison entre les droites et les sphères. Proposons-nous de trouver toutes les transformations de l'espace ordinaire qui changent les lignes droites en sphères. Dans le cas de trois variables $x, y, z$ il y aura trois classes de transformation de contact suivant qu'on établit un, deux ou trois rélations entre $x, y, z, X, Y, Z$. Nous sommes donc conduits à déterminer les formes d'un, deux ou trois équations directrices qui sont capables de représenter droite-en-sphère transformations de contact.

Supposons qu'on parte d'une seule relation entre $x, y, z, X, Y, Z$

$$
\Phi(x, y, z, X, Y, Z)=0 \text {. }
$$

La droite

$$
x=a z+b, \quad y=c z+d
$$


sera transformée dans la surface donnée par l'équation

$$
\Phi(X, Y, Z, a, b, c, d)=0
$$

que l'on obtient en éliminant $z$ au moyen des équations

$$
\Phi(a z+b, c z+d, \quad z, \quad X, Y, Z)=0, \quad \Phi_{z}=0 .
$$

Pour que la transformation soit unique, il est clair que le degré de la fonction $\Phi$ par rapport au variable $z$ doit être égal deux au plus; c'est-à-dire, la fonction $\Phi$ doit être de la forme

$$
\Phi_{1} x^{2}+\Phi_{2} y^{2}+\Phi_{3} z^{2}+\Phi_{4} y z+\Phi_{5} z x+\Phi_{6} x y+\Phi_{7} x+\Phi_{8} y+\Phi_{9} z+\Phi_{10}=0,
$$

où les $\Phi_{i}$ sont des fonctions des variables $X, Y, Z$. Ainsi, en posant

$$
P=a^{2} \Phi_{1}+c^{2} \Phi_{2}+\Phi_{3}+c \Phi_{4}+a \Phi_{5}+a c \Phi_{6},
$$

$Q=2 a b \Phi_{1}+2 c d \Phi_{2}+d \Phi_{4}+b \Phi_{5}+(a d+b c) \Phi_{8}+a \Phi_{7}+c \Phi_{s}+\Phi_{9}$,

$\boldsymbol{R}=b^{2} \Phi_{1}+d^{2} \Phi_{2}+b d \Phi_{6}+b \Phi_{7}+d \Phi_{8}+\Phi_{10}$,

la transformée de la droite (2) est la surface

ou

$$
Q^{2}-4 P R=0
$$

$\left.d^{2} \Phi_{4}^{2}+b\right) \Phi_{5}^{2}+(a d-b c)^{2} \Phi_{6}^{\prime}+a^{2} \Phi_{7}^{2}+c^{2} \Phi_{8}^{2}+\Phi_{9}^{2}$

$-4 \Phi_{1}\left\{(a d-b c)^{2} \Phi_{2}+b^{2} \Phi_{3}-b(a d-b c) \Phi_{4}\right.$

$$
+a(a d-b c) \Phi_{8}-a d \Phi_{9}+a^{2} \Phi_{10} !
$$

$-4 \Phi_{2}\left\{d^{2} \Phi_{3}+d(a d-b c) \Phi_{5}-c(a d-b c) \Phi_{7}-c d \Phi_{8}+c^{2} \Phi_{10}\right\}$

$-4 \Phi_{3}\left|b d \Phi_{6}+b \Phi_{7}+d \Phi_{8}+\Phi_{10}\right|$

$+2 \Phi_{4} \mid b d \Phi_{5}+d(a d-b c) \Phi_{6}+(a d-2 b c) \Phi_{7}$

$$
-c d \Phi_{8}+d \Phi_{9}-c \Phi_{10}
$$

$-2 \Phi_{5} \mid b(a d-b c) \Phi_{6}+a b \Phi_{7}+(2 a d-b c) \Phi_{8}-b \Phi_{9}+2 a \Phi_{10}$

$-2 \Phi_{6}\left|a(a d-b c) \Phi_{7}+c(a d-b c) \Phi_{8}+(a d+b c) \Phi_{9}-2 a c \Phi_{19}\right|$

$\left.+2 \Phi_{7} \mid a c \Phi_{8}+a \Phi_{9}\right\}+2 c \Phi_{8} \Phi_{9}=0$.

Pour que cette surface soit une quadrique pour toutes les valeurs de $a, b, c, d$ il faut que les fonctions $\Phi_{i}$ aient la forme

$$
\Phi_{i}=l_{i} X+m_{i} Y+n_{i} Z+\mu_{i}, \quad i=1,2, \ldots, 10,
$$

où les $l_{i}, m_{i}, n_{i}, \mu_{i}$ sont des constantes.

Annati di Matematica, Serie III, tomo VII 
Si l'on veut que cette quadrique soit une sphère pour toutes les valeurs, de $a, b, c, d$, il est nécessaire que les fonctions

$$
\Phi_{4}, \Phi_{5}, \Phi_{8}, \Phi_{7}, \Phi_{8}, \Phi_{9}
$$

se réduisent à des constantes absolues. Donc en introduisant les hypothèses suivantes qui n'imposent aucune restriction

$$
\Phi_{4}=\Phi_{5}=\Phi_{6}=\Phi_{7}=\Phi_{8}=\Phi_{9}=0,
$$

l'équation de la quadrique devient

$$
(a d-b c)^{2} \Phi_{1} \Phi_{2}+d^{2} \Phi_{2} \Phi_{3}+b^{2} \Phi_{3} \Phi_{1}+a^{2} \Phi_{3} \Phi_{10}+c^{2} \Phi_{2} \Phi_{10}+\Phi_{3} \Phi_{10}=0 .
$$

Pour que cette surface-ci soit une sphère il faut et il suffit qu'on ait les relations suivantes

$$
\begin{gathered}
S l_{1} l_{2}=S l_{2} l_{3}=S l_{3} l_{1}=S l_{1} l_{10}=S l_{2} l_{10}=S l_{3} l_{10}, \\
l_{1} m_{2}-l_{2} m_{1}=l_{1} m_{3}-l_{3} m_{1}=\cdots=l_{1} n_{2}-l_{2} n_{1}=\cdots=m_{1} n_{10}-m_{10} n_{1}=0 .
\end{gathered}
$$

Les équations (16) sont équivalentes aux relations

$$
l_{1}: l_{2}: l_{3}: l_{10}=m_{1}: m_{2}: m_{3}: m_{10}=n_{1}: n_{2}: n_{3}: n_{10}
$$

par conséquent

$$
\begin{gathered}
\Psi_{1}=\lambda+\beta Y+\gamma Z, \quad \Psi_{i}=k_{i} \Psi_{1}, \quad i=2,3, \ldots, 10, \\
\Psi_{j}=\Phi_{i}-\mu_{j}, \quad j=1,2, \ldots, 10,
\end{gathered}
$$

et, en vertu des équations (15), on a

c'est-à-dire

$$
k_{2}=k_{3}=k_{10}=1 ;
$$

$$
\Phi_{i}=\alpha X+\beta Y+\gamma Z+\mu_{i}, \quad i=1,2, \ldots, 10 .
$$

Ainsi, la forme de l'équation directrice devient

$$
\Phi=(\alpha X+\beta Y+\gamma Z)\left(x^{2}+y^{2}+z^{2}+1\right)+\mu_{1} x^{2}+\mu_{2} y^{2}+\mu_{3} x^{2}+\mu_{4}=0 .
$$

Il faudra adjoindre à cette équation pour déterminer $X, Y, Z, P, Q$ les équations suivantes

$$
\Phi_{x}+p \Phi_{z}=0, \quad \Phi_{4}+q \Phi_{z}=0, \quad \Phi_{x}+P \Phi_{z}=0, \quad \Phi_{4}+Q \Phi_{z}=0 ;
$$

done on trouve la forme explicite de la droite-en-sphère transformation de contact au moyen de la résolution par rapport aux $X, Y, Z, P, Q$ du 
système :

$$
\begin{gathered}
(x+p z)(\alpha X+\beta Y+\gamma Z)+\mu_{1} x+\mu_{3} p=0 \\
(y+q z)(\alpha X+\beta Y+\gamma Z)+\mu_{2} y+\mu_{3} q=0 \\
\left(x^{2}+y^{2}+z^{2}+1\right)(\alpha X+\beta Y+\gamma Z)+\mu_{1} x^{2}+\mu_{2} y^{2}+\mu_{3} z^{2}+\mu_{4}=0 \\
\gamma P+\alpha=0, \quad \gamma Q+\beta=0 .
\end{gathered}
$$

Mais il n'est pas possible de résoudre ce système-ci par rapport aux $X$, $Y, Z$ et ainsi, la fonction $\Phi(21)$ n'est pas capable de définir une transformation de contact. On conclut de là qu'jl n'y a point de transformations de contact univoques déterminées par une équation directrice, qui changent les lignes droites en sphères.

On remarque, en passant, que toutes les $\infty^{\infty}$ transformations de contact définies par une équation directrice de la forme

$x \rho_{1}(X, Y, Z)+y \rho_{2}(X, Y, Z)+z \rho_{3}(X, Y, Z)+\rho_{4}(X, Y, Z)=0$,

transforment les lignes droites en des lignes courbes; que toutes les $\infty^{\infty}$ transformations définies par une équation de la forme

$$
\sigma_{1}(x, y, z) X+\sigma_{2}(x, y, z) Y+\sigma_{3}(x, y, z) Z+\sigma_{4}(x, y, z)=0
$$

changent les lignes droites en des surfaces développables; et enfin, que les seules transformations de contact définies par une équation qui transforment les lignes droites en des lignes droites sont les $\infty^{15}$ transformations déterminées par l'équation bilinéaire

$$
\left(l_{1} X+m_{1} Y+n_{1} Z+\mu_{1}\right) x+\cdots=0 .
$$

Les transformations (25) sont équivalentes aux transformations ponctuelles arbitraires

$$
x_{1}=\sigma_{1}(x, y, z)\left|\sigma_{4}(x, y, z), \ldots, z_{1}=\sigma_{3}(x, y, z)\right| \sigma_{1}(x, y, z)
$$

suivies de la transformation par polaires réciproques par rapport à la sphère

$$
X^{2}+Y^{2}+Z^{2}-1=0
$$

déterminée par l'équation directrice

$$
X x_{1}+Y y_{1}+Z \approx_{s_{1}}-1=0 \text {. }
$$

Dans le cas des transformations (26) les transformations (27) sont des transformations homographiques. 
Supposons maintenant qu'il existe deux relations indépendantes entre $x, y, z, X, Y, Z$ :

$$
\Omega_{1}(x, y, z, X, Y, Z)=0, \quad \Omega_{2}(x, y, z, X, Y, Z)=0 .
$$

La transformation la plus générale déterminée par ces deux équations directrices changent la ligne droite

$$
y+k x+m=0, \quad z+l x+n=0,
$$

en la surface donnée par l'équation

$$
\Omega(X, Y, Z, k, l, m, n)=0,
$$

que l'on obtient en éliminant $x, y, z$ au moyen des quatre équations (30) et (31).

Pour que la ligne droite soit transformée dans une surface unique il faut que l'une des équations (30) soit linéaire par rapport aux variables $x$, $y, z$; pour que la transformée soit une quadrique il est nécessaire que l'autre équation soit linéaire dans $x, y, z$ et que les deux équations soient linéaires par rapport aux variables $X, Y, Z$.

Considérons done les $\infty^{33}$ transformations qui sont déterminées par deux équations bilinéaires

où

$$
x \varphi_{1}+y \varphi_{2}+z \varphi_{3}+\varphi_{4}=0, \quad x \varphi_{5}+y \varphi_{6}+z \varphi_{7}+\varphi_{8}=0,
$$

$$
\varsigma_{i} \equiv a_{i} X+b_{i} Y+c_{i} Z+d_{i} .
$$

La transformée de la ligne droite (31) sera la surface du second degré

$$
\begin{gathered}
\left|\begin{array}{ll}
k & l \\
m & n
\end{array}\right| \cdot\left|\begin{array}{ll}
\varphi_{2} & \varphi_{3} \\
\varphi_{6} & \varphi_{7}
\end{array}\right| \\
-k\left|\begin{array}{ll}
\varphi_{2} & \varphi_{4} \\
\varphi_{6} & \varphi_{8}
\end{array}\right|-l\left|\begin{array}{ll}
\varphi_{3} & \varphi_{4} \\
\varphi_{7} & \varphi_{8}
\end{array}\right|-m\left|\begin{array}{ll}
\varphi_{1} & \varphi_{8} \\
\varphi_{5} & \varphi_{6}
\end{array}\right|-\mid \\
-n\left|\begin{array}{ll}
\varphi_{1} & \varphi_{3} \\
\varphi_{5} & \varphi_{7}
\end{array}\right|+\left|\begin{array}{ll}
\varphi_{1} & \varphi_{4} \\
\varphi_{5} & \varphi_{8}
\end{array}\right|=0 .
\end{gathered}
$$

Cette quadrique peut se réduire à une sphère pour toutes les valeurs de $k, l, m, n$ dans les deux cas suivants:

1." Quand tous les six déterminants de la matrice

$$
\left\|\begin{array}{llll}
\psi_{1} & \psi_{2} & \psi_{3} & \psi_{4} \\
\psi_{5} & \psi_{6} & \psi_{7} & \psi_{8}
\end{array}\right\| \quad \psi_{i} \equiv \varphi_{i}-d_{i}
$$

se réduisent à la forme

$$
\text { Const. }\left(X^{2}+Y^{2}+Z^{2}\right)
$$


2. ${ }^{\circ}$ Quand un détérminant quelconque de la matrice se réduit à la forme (37) et les fonctions $\varphi_{i}$ correspondant aux fonctions $\psi_{i}$ restant dans la matrice se réduisent à des constantes: par exemple, soient les fonctions $\varphi_{2}$, $\varphi_{3}, \varphi_{6}, \varphi_{7}$ constantes et le déterminant $\varphi_{1} \psi_{8}-\varphi_{4} \psi_{5}$ de la forme (37).

Dans le premier eas nous avons deux familles de $\boldsymbol{\infty}^{15}$ droite-en-sphère transformations de contact.

En effet les équations

$$
\begin{aligned}
& a_{i} a_{\sigma}-a_{\rho} a_{j}=b_{i} b_{\sigma}-b_{\rho} b_{j}=c_{i} c_{\sigma}-c_{\rho} c_{j}=1=0 \\
& a_{i} b_{\sigma}+b_{i} a_{\sigma}-a_{\rho} b_{j}-b_{\rho} a_{j}=0 \\
& b_{i} c_{\sigma}+c_{i} b_{\sigma}-b_{\rho} c_{j}-c_{\rho} b_{j}=0 \\
& c_{i} a_{\sigma}+a_{i} c_{\sigma}-c_{\rho} a_{j}-a_{\rho} c_{j}=0
\end{aligned}
$$

qui sont nécessaires et suffisantes pour que le déterminant

$$
\psi_{i} \psi_{\sigma}-\psi_{p} \psi_{j}
$$

soit changé dans la forme (37) possédant les solutions symétriques

$$
\begin{aligned}
& b_{i}=a_{i} \sqrt{-1}, \quad b_{\rho}=-a_{\rho} \sqrt{-1}, \quad b_{j}=a_{j} \sqrt{-1}, \quad b_{\sigma}=-a_{\sigma} \sqrt{-1} \\
& c_{i}= \pm a_{\beta}, \quad c_{\beta}=\mp a_{i} \\
& c_{j}= \pm a_{\sigma}, \quad c_{\sigma}=\mp a_{j}
\end{aligned}
$$

ainsi, on trouve que les deux systèmes de fonctions

$$
\begin{gathered}
\varphi_{j}=a_{j} X+i a_{j} Y+c_{j} Z+d_{j}, \\
\varphi_{j+4}=c_{j} X-i c_{j} Y-a_{j} Z+d_{j+4}, \quad j=1,2,3,4 ; \\
\varphi_{j}^{\prime}=a_{j} X+i a_{j} Y-c_{j} Z+d_{j}, \\
\xi^{\prime}{ }_{j+4}=c_{j} X-i c_{j} Y+a_{j} Z+d_{j+4}=0, \quad j=1,2,3,4 ;
\end{gathered}
$$

sont capables de définir deux familles de $\infty^{\text {ts }}$ transformations qui changent les lignes droites en sphères.

En formant les équations

$$
\begin{aligned}
& \Omega_{1}=\underbrace{4}_{1}\left(a_{i j} X+i a_{1 j} Y+a_{2 j} Z+a_{3 j}\right) x_{j}=0, \\
& \quad x_{1}=x, x_{2}=y, x_{3}=z, x_{4}=1, i=\sqrt{-1}, \\
& \Omega_{2}=\sum_{1}^{4}\left(a_{3 j} X-i a_{2 j} Y-a_{1 j} Z+a_{4 j}\right) x_{j}=0, \\
& \frac{\Omega_{1}^{x}}{\Omega_{3}^{x}}=\frac{\Omega_{1}^{y}}{\Omega_{2}^{y}}=\frac{\Omega_{1}^{X}}{\Omega_{i}^{X}}=\frac{\Omega_{1}^{Y}}{\Omega_{q}^{Y}},
\end{aligned}
$$


et les équations

où

$$
\begin{gathered}
\Omega_{3}=\sum_{1}^{4} \mathbf{y}_{j}\left(a_{1 j} X-i a_{i j} Y+a_{2 j} Z+a_{3 j}\right) x_{j}=0, \\
\Omega_{1}=\sum_{1}^{4}\left(a_{2 j} X+i a_{2 j} Y-a_{1 j} Z+a_{i j}\right) x_{j}=0, \\
\frac{\Omega_{3}^{x}}{\Omega_{4}^{x}}=\frac{\Omega_{3}^{y}}{\Omega_{4}^{y}}=\frac{\Omega_{3}^{X}}{\Omega_{4}^{X}}=\frac{\Omega_{3}^{Y}}{\Omega_{4}^{Y}},
\end{gathered}
$$

$$
\begin{gathered}
\omega^{x}=\omega_{x}+p \omega_{z}, \quad \omega^{y}=\omega_{y}+q \omega_{z}, \\
\omega^{X}=\omega_{X}+P \omega_{Z}, \quad \omega^{Y}=\omega_{Y}+Q \omega_{Z},
\end{gathered}
$$

nous vérifions facilement que les transformations définies par les équations dérivées sont des transformations de contact. Nous voyons ensuite que, en particularisant les constantes des équations (43) de la manière suivante

$$
\begin{gathered}
a_{14}=a_{21}=a_{33}=a_{42}=1, \\
a_{14}=a_{12}=a_{13}=a_{22}=a_{23}=a_{24}=a_{31}=a_{32}=a_{34}=a_{11}=a_{43}=a_{44}=0 ;
\end{gathered}
$$

ou les constantes des équations (44) de la manière suivante

$$
\begin{gathered}
a_{11}=-a_{24}=a_{32}=-a_{43}=1, \\
a_{12}=a_{13}=a_{14}=a_{21}=a_{22}=a_{23}=a_{31}=a_{33}=a_{34}=a_{41}=a_{42}=a_{44}=0,
\end{gathered}
$$

nous avons la correspondance célèbre étudiée par LIE

$$
Z x_{1}+z_{1}+X+i Y=0, \quad(X-i Y) x_{1}+y_{1}-Z=0 .
$$

En combinant la transformation de Lif (48) avec toutes les droite-endroite transformations ponctuelles $d u$ groupe projectif général

$$
\left.\begin{array}{l}
\rho x_{1}=\alpha_{1} x+\beta_{1} y+\gamma_{1} z+\delta_{1}, \quad \rho z_{1}=\alpha_{3} x+\beta_{3} y+\dot{\gamma}_{3} z+\delta_{3}, \\
\rho y_{1}=\alpha_{2} x+\beta_{2} y+\gamma_{2} z+\delta_{2}, \quad \rho=\alpha_{4} x+\beta_{4} y+\gamma_{4} z+\delta_{4},
\end{array}\right\}
$$

on obtient $\infty^{15}$ droite-en-sphère transformations de contact déterminées par deux équations

$$
\left.\begin{array}{l}
\left(\alpha_{4} X+i \alpha_{4} Y+\alpha_{1} Z+\alpha_{3}\right) x+\cdots=0, \\
\left(\alpha_{1} X-i \alpha_{1} Y-\alpha_{4} Z+\alpha_{2}\right) x+\cdots=0,
\end{array}\right)
$$

qui sont de la forme (43). 
Donc les transformations $(S)$ de la famille (43) se déduisent de la transformation $(\Lambda)$ de Lre (48) et des transformations (I) de groupe projectif général au moyen de l'équation symbolique

$$
S=\text { II } \Lambda \text {. }
$$

Encore on combine la transformation de LiE avec toutes les droite-endroite transformations de contact définies par l'équation

$$
\left(\alpha_{1} X+\beta_{1} Y+\gamma_{1} Z+\delta_{1}\right) x+\cdots=0 ;
$$

les $\infty^{15}$ droite-en-sphère transformations $(\Sigma)$ résultantes sont déterminées par les deux équations

$$
\begin{aligned}
& \left(\alpha_{1} X-i \alpha_{1} Y+\alpha_{4} Z+\alpha_{2}\right)+\cdots=0, \\
& \left(\alpha_{4} X+i \alpha_{4} Y-\alpha_{1} Z+\alpha_{3}\right)+\cdots=0,
\end{aligned}
$$

de la forme (44); ainsi pour les transformations (44) on a l'équation symbolique

$$
\Sigma=D \Lambda,
$$

où $D$ est la transformation dualistique générale.

Revenons maintenant au deuxième cas, savoir quand un déterminant quelconque de la matrice (36) se réduit à la forme (37) et les fonctions ți correspondant une fonction $\psi_{i}$ restant dans la matrice se réduisent a des constantes. Nous trouvons six familles de $\infty^{13}$ transformations définies par deux équations bilinéaires de la forme

$$
\begin{aligned}
& \omega_{5}=\left(a_{1} X+b_{4} Y+c_{4} Z+d_{1}\right) x+ \\
& \quad+d_{2} y+d_{3} z+a_{4} X+b_{4} Y+c_{4} Z+d_{4}=0 \\
& \omega_{5}=\left(a_{5} X+b_{5} Y+c_{5} Z+d_{5}\right) x+ \\
& +d_{6} y+d_{7} z+a_{8} X+b_{8} Y+c_{8} Z+d_{8}=0
\end{aligned}
$$

où les constantes sont assujetties aux conditions

$$
\begin{aligned}
& a_{1} a_{8}-a_{4} a_{5}=b_{1} b_{8}-b_{4} b_{5}=c_{4} c_{8}-c_{4} c_{8}=1=0 \\
& a_{1} b_{8}+a_{8} b_{1}-a_{4} b_{5}-a_{5} b_{4}=0 \\
& b_{1} c_{8}+b_{8} c_{4}-b_{4} c_{5}-b_{5} c_{4}=0 \\
& c_{1} a_{8}+c_{8} a_{4}-c_{4} a_{5}-c_{5} a_{4}=0 .
\end{aligned}
$$


Des équations

$$
\left.\begin{array}{c}
\omega_{5}=0, \quad \omega_{6}=0, \\
\frac{\omega_{5}^{x}}{\omega_{6}^{x}}=\frac{\omega_{5}^{y}}{\omega_{6}^{y}}=\frac{\omega_{5}^{X}}{\omega_{6}^{X}}=\frac{\omega_{5}^{X}}{\omega_{6}^{Y}}
\end{array}\right\}
$$

nous voyons que les transformations sont des transformations de contact.

Les droites se transforment en des points si

$$
d_{1}: d_{2}: d_{3}: d_{4}=d_{5}: d_{6}: d_{7}: d_{8}
$$

et en des plans, si

$$
a_{1}: b_{1}: c_{1}: a_{4}: b_{4}: c_{4}=a_{5}: b_{5}: c_{5}: a_{8}: b_{8}: c_{8} .
$$

Nous voyons ensuite que, en particularisant les constantes de la manière suivante

$$
\left.\begin{array}{l}
a_{4}=a_{5}=c_{1}=-c_{8}=d_{3}=d_{6}=1, \quad b_{4}=-b_{5}=\sqrt{-1}, \\
a_{1}=a_{8}=b_{1}=b_{8}=c_{4}=c_{5}=d_{1}=d_{2}=d_{5}=d_{7}=d_{8}=0
\end{array}\right\}
$$

nous avons la transformation (48) de Lie.

Enfin les valeurs des constantes

$$
\begin{aligned}
& a_{3}=a_{6}=-a_{8}=-c_{2}=c_{7}=d_{4}=1, \quad b_{3}=-b_{6}=\sqrt{-1} \\
& \begin{aligned}
a_{1}=a_{4}=a_{5}=b_{1}=b_{4} & =b_{5}=c_{1}=c_{4}=c_{5}=c_{8}= \\
& =d_{1}=d_{2}=d_{5}=d_{6}=d_{7}=d_{8}=0
\end{aligned}
\end{aligned}
$$

donnent la transformation de contact définie par les équations

$$
(X+i Y) z-Z y+1=0, \quad(X-i Y) y+Z z-x=0
$$

cette transformation est équivalente à une transformation par polaires réciproques détèrminée par l'équation directrice

$$
X x_{1}+Y y_{1}+Z z_{1}-1=0
$$

suivie de la transformation de Lie.

Pour obtenir les formes explicites des transformations de cette famille (55) et (56) on fait un changement des constantes arbitraires et on considérait la forme quadratique

$$
\left|\begin{array}{ll}
1_{1} X+1_{2} Y+1_{3} Z & 4_{1} X+4_{2} Y+4_{3} Z \\
3_{1} X+3_{2} Y+3_{3} Z & 2_{1} X+2_{2} Y+2_{3} Z
\end{array}\right|
$$


qui se réduit à la forme

$$
\text { Const. }\left(X^{2}+Y^{2}+Z^{2}\right)
$$

si les constantes soient assujetties aux conditions:

$$
\begin{aligned}
& 1_{1} 2_{1}-3_{1} 4_{1}=1_{2} 2_{2}-3_{2} 4_{2}=1_{3} 2_{3}-3_{3} 4_{2}==0 \\
& 1_{1} 2_{2}+1_{2} 2_{1}-34_{2}-3_{2} 4_{1}=0 \\
& 1_{2} 2_{3}+1_{3} 2_{2}-3_{2} 4_{3}-3_{3} 4_{3}=0 \\
& 1_{3} 2_{1}+1_{1} 3_{2}-3_{3} 4_{1}-3_{1} 4_{3}=0
\end{aligned}
$$

A cause de la symétrie et de l'invariance de ces équations-ci sous les substitutions

$$
(1234):(2143) ; \quad(1234):(4321) ; \quad(1234):(3412) ;
$$

et sous une permutation cyclique des indices, il est nécessaire à considérer seulement les quarante cinq cas suivants des sept cent quatre-vingt douze manières à choisir sept constantes arbitraires:

$10^{\circ}$ cas : Soient les quantités

$$
1_{1}, 1_{2}, 1_{3}, 3_{1}, 4_{1}, 4_{2}, 4_{3}
$$

des constantes tout-à-fait arbitraires; les équations de condition sont linéaires et les cinq constantes restantes sont des fonctions suivantes des constantes données

$$
2_{1}=3_{1} 4_{1} \Delta, \quad 2_{2}=3,4_{2} \Delta, \quad 2_{3}=3_{1} 4_{3} \Delta, \quad 3_{2}=3_{1} 1_{2} \Delta, \quad 3_{3}=3_{1} 1_{3} \Delta,
$$

où

$$
\Delta=\left|\begin{array}{ccccc}
0 & 4_{1} & -1_{3} & 0 & -1 \\
4_{1} & 0 & -1_{2} & 1_{1} & 0 \\
4_{2} & 0 & 1_{1} & -1_{2} & 0 \\
4_{2} & -4_{3} & 0 & 1_{2} & 1_{3} / 1_{1} \\
4_{3} & 4_{2} & 0 & -1_{3} & -1_{2} / 1_{1}
\end{array}\right|
$$

2. ${ }^{\circ}$ cas: Supposons que les constantes

$$
1_{1}, 1_{2}, 1_{3}, 3_{1}, 3_{2}, 3_{3}, 4_{1}
$$

aient des valeurs arbitraires; on construit immédiatement les formes des inconnues en remarquant que ce cas-ci se déduit du premier cas par la sub- 
stitution

$$
\left(3,3_{2}, 3_{3} 4_{1} 4_{2} 4_{3}\right):\left(4,4_{2} 4_{3} 3_{1} 3_{2} 3_{3}\right)
$$

3. ${ }^{\circ}$ cas : Sojent

$$
1_{1}, 1_{2}, 1_{3}, 2_{1}, 2_{2}, 2_{3}, 4_{1}
$$

les données; en posant

$$
\begin{array}{lll}
\alpha=1_{1} 2_{2}+1_{2} 2_{1}, & \beta=1_{2} 2_{3}+1_{3} 2_{2}, & \gamma=1_{2} 2_{1}+1_{1} 2_{3}, \\
\delta=1_{2} 2_{2}-1,2_{1}, & \varepsilon=1_{2} 2_{2}-1_{3} 2_{3}, & \zeta=4_{1},
\end{array}
$$

on trouve

$$
\begin{aligned}
& 3_{1}=\frac{\alpha \zeta-\delta \zeta}{\xi^{2}+\zeta^{2}}, \quad 3_{2}=\frac{\alpha \zeta+\delta \xi}{\zeta^{2}+\zeta^{2}}, \\
& 3_{2}=\frac{\gamma(\alpha \zeta+\delta \xi)-\beta(\alpha \zeta-\delta \zeta)}{\zeta(\alpha \zeta+\delta \xi)-\xi(\alpha \xi-\delta \zeta)}, \quad 4_{3}=\frac{(\beta \zeta-\gamma \zeta)\left(\zeta^{2}+\zeta^{2}\right)}{\alpha \zeta^{2}+2 \delta \xi \zeta-\alpha \xi_{2}^{2}},
\end{aligned}
$$

où $\xi \equiv 4_{2}$ est unc racine de l'équation du sixième degré

$$
\rho_{0} x^{6}+\zeta \alpha \rho_{1} x^{5}+\zeta^{2} \rho_{2} x^{4}+\zeta^{3} \alpha \rho_{3} x^{3}+\zeta^{4} \rho_{4} x^{2}+\zeta^{5} \alpha \rho_{5} x+\zeta^{6} \rho_{6}=0,
$$

où

$$
\begin{gathered}
\rho_{0}=\alpha^{2}(\delta-\varepsilon)-\gamma(\alpha \beta-\gamma j), \quad \rho_{1}=\alpha^{2}+\beta^{2}+\gamma^{2}-4 \delta(\delta-\varepsilon), \\
\rho_{2}=\alpha^{2}(\varepsilon-6 \delta)-3 \alpha \beta \gamma-\left(\beta^{2}-2 \gamma^{2}\right) j+4 \delta^{2}(j-\varepsilon), \\
\rho_{3}=2\left(\beta^{2}+\gamma^{2}-\alpha^{2}+4 \delta^{2}\right), \quad \rho_{4}=\alpha^{2}(\varepsilon+5 \delta)-3 \alpha \beta \gamma-\left(2 \beta^{2}-\gamma^{2}\right) \delta-4 \delta^{2} \varepsilon, \\
\rho_{5}=\alpha^{2}+\gamma^{2}-\beta^{2}-3 \delta \varepsilon, \quad \rho_{0}=-\alpha^{2} \varepsilon-\beta^{2} \delta-\alpha \beta \gamma .
\end{gathered}
$$

4. ${ }^{\circ}$ cas: Les données sont

$$
1_{1}, 1_{z}, 1_{3}, 2_{1}, 2_{2}, 3_{1}, 3_{z}
$$

nous avons

$$
4_{1}=\frac{1_{1} 2_{1} 3_{1}-1_{2} 2_{2} 3_{1}+1_{1} 2_{1} 3_{2}}{3_{1}^{2}+3_{2}^{2}}, \quad 4_{2}=\frac{1_{1} 2_{1} 3_{1}-1_{1} 2_{1} 3_{2}+1_{2} 2_{2} 3_{2}}{3_{1}^{2}+3_{2}^{2}},
$$

la quantité $4_{3}$ est une racine de l'équation quadratique

$$
l x^{2}+m x+n-0
$$

où

$$
\begin{aligned}
& l=1_{2} 1_{3}\left(3_{1}^{2}+3^{2}\right)\left(1_{1} 3_{2}-1_{2} 3_{1}\right), \\
& \left.m=\left(3_{1}^{2}+3_{2}^{2}\right): 1_{2}^{2} 1_{3}^{2}\left(3_{1}^{2} 2_{1}+22_{2} 3_{3} 3_{2}+3_{2}^{2} 2_{1}\right)-21_{1} 1_{2} 1_{3}^{2}\left(2,3_{1} 3_{2}+2_{2} 3_{2}\right)\right\} \text {, }
\end{aligned}
$$




$$
\begin{gathered}
n=1_{113}\left\{\left(1_{12} 2_{11}-1_{33} 2_{12}\right)\left(3_{111}-3_{112}+3_{122}-3_{222}\right)\right\} \\
-1_{123}\left\{\left(1_{12} 2_{11}-1_{33} 2_{12}\right)\left(3_{111}+3_{112}+3_{122}-3_{322}\right)+\right. \\
+1_{12} 2_{12}\left(3_{111}-33_{112}-3_{122}-3_{222}\right)+ \\
\left.+2_{22}\left(1_{22}+1_{33}\right) 3_{112}+1_{11} 2_{11} 3_{122}+1_{22} 1_{33} 3_{222}\right\} \\
-1_{223}\left\{\left(1_{22} 2_{22}-31_{12} 2_{12}+1_{33} 2_{22}\right) 3_{114}+21_{11} 2_{11} 3_{142}+\right. \\
\left.+\left(1_{11} 2_{11}-1_{12} 2_{12}+1_{33} 2_{22}\right) 3_{122}\right\} \\
-1_{333}\left\{\left(1_{12} 2_{11}-1_{11} 2_{12}\right)\left(3_{111}-3_{112}+3_{122}-3_{223}\right)+\right. \\
\left.+\left(1_{22} 2_{12}-1_{12} 2_{22}\right)\left(3_{112}+3_{222}\right)\right\} \\
k_{l m n}=k_{l} k_{m} k_{n}
\end{gathered}
$$

enfin les paramètres

$$
2_{3}=\theta, \quad 3_{3}=\varphi
$$

sont données par les équations

où

$$
\begin{gathered}
\left(1_{23} \xi-1_{31} \eta\right) \varphi=1_{33}\left(1_{2} 2_{1}-1_{1} 2_{2}\right)+\left(1_{13} 3_{2}-1_{23} 3_{1}\right) \zeta \\
1_{1} \theta=3_{1} \zeta+\xi \varphi+1_{3} 2_{1}
\end{gathered}
$$

$$
\xi=4_{1}, \quad \eta=4_{2}, \quad \zeta=4_{3} .
$$

$5 .^{\circ}$ cas: On cherche les valeurs des constantes

$$
2_{1}, 2_{2}, 2_{3}, 3_{3}, 4_{3}
$$

comme fonctions des constantes

on trouve

$$
1_{1}, 1_{2}, 1_{3}, 3_{1}, 3_{2}, 4_{1}, 4_{2} ;
$$

$$
\begin{aligned}
& 2_{1}=\zeta=\frac{1_{1}\left(3_{1} 4_{1}-3_{2} 4_{2}\right)+1_{2}\left(3_{1} 4_{2}+3_{2} 4_{1}\right)}{1_{1}^{2}+1_{2}^{2}}, \\
& 2_{2}=\eta=\frac{1_{1}\left(3_{1} 4_{2}+3_{2} 4_{1}\right)--1_{2}\left(3_{1} 4_{1}-3_{2} 4_{2}\right)}{1_{1}^{2}+1_{2}^{2}},
\end{aligned}
$$

en substituant ces valeurs dans les équations

$$
\begin{aligned}
& \left(3_{1} 4_{2}-3_{2} 4_{1}\right) \theta=\left|\begin{array}{ll}
1_{2} \zeta+1_{3} \eta & 3_{2} \\
1_{1} \zeta+1_{3} \zeta & 3_{4}
\end{array}\right|, \\
& \left(3_{1} 4_{2}-3_{2} 4_{1}\right) \varphi=\left|\begin{array}{ll}
4_{2} & 1_{2} \zeta+1_{3} \eta \\
4_{1} & 1_{1} \zeta+1_{3} \xi
\end{array}\right|,
\end{aligned}
$$


on a $\vartheta=3_{3}$, et $\xi=4_{3}$ en des fonctions de $\zeta=2_{3} ;$ enfin l'équation

$$
\varphi \theta+1_{2} \eta-1_{3} \zeta-3_{2} 4_{2}=0
$$

devient une équation quadratique en $\zeta$

où

$$
\lambda \zeta^{2}+\mu \zeta+\nu=0
$$

$$
\begin{array}{r}
\lambda=\left(1_{2} 3_{1}-1,3_{2}\right)\left(1_{1} 4_{2}-1_{2} 4_{1}\right), \quad \nu=\left(1_{2} \eta-3_{2} 4_{2}\right)\left(34_{2}-3_{2} 4_{1}\right)^{2} \\
\mu=\left(1_{3} 4_{2} \xi-1_{3} 4_{1} \eta\right)\left(1_{2} 3_{1}-1_{1} 3_{3}\right)-\left(1_{1} 4_{2}-1_{2} 4_{1}\right)\left(1_{3} 3_{2} \xi-1_{3} 3_{1} \eta\right)- \\
-1_{3}\left(3_{1} 4_{2}-3_{2} 4_{1}\right)^{2}
\end{array}
$$

6." cas : Pour déterminer

$$
\xi=3_{1}, \quad \eta=3_{2}, \quad \zeta=3_{3}, \quad \theta=4_{3}, \quad \varphi=2_{3}
$$

en fonctions des autres coefficients il suffit d'observer que ce cas-ci se déduit du cas $4 .^{\circ}$ par la substitution

$$
\left(\theta \varphi 3_{1} 3_{2}\right):\left(\varphi \theta 4_{1} 4_{2}\right) .
$$

7." cas: Il s'agit de trouver

$$
\xi=4_{1}, \quad \eta=4_{2}, \quad \zeta=4, \quad \Leftrightarrow=2_{3}, \quad,=3_{1}
$$

en termes des autres constantes; il parait que ost une racine de la cubique

où

$$
a \dot{\varphi}^{3}+b \varphi^{2}+c \varphi+d=0
$$

$$
\begin{aligned}
& a=2_{2} 3_{2}\left(1_{22}+1_{33}\right), \quad b=-3_{2}\left\{2_{1} 3_{2}\left(1_{22}+1_{33}\right)+21_{12} 2_{2} 3_{2}+21_{13} 2_{2} 3_{3}\right\} \\
& c=3_{222}\left(1_{11} 2_{2}+1_{1} 2_{1}\right)+3_{23}\left(1_{13} 2_{1} 2_{3}-1_{23} 2_{2} 3_{3}-1_{12} 2_{2}-1_{22} 2_{1}\right)+ \\
& +3_{2}\left(1_{11} 2_{2} 3_{33}+1_{22} 2_{2} 3_{33}-i_{13} 2_{2} 3_{3}-1_{23} 2_{1} 3_{3}\right) \\
& d=1_{3} 2_{1} 3_{292}\left(1_{2} 3_{3}-1_{3} 2_{2}\right)+1_{1} 3_{23}\left(3_{22}+3_{33}\right)\left(1_{2} 2_{2}-1_{1} 2_{1}\right)- \\
& -3_{22} 3_{3}\left(1_{1} 2_{2}+1_{2} 2_{1}\right)\left(1_{2} 3_{3}-1_{3} 3_{2}\right)- \\
& -3_{22}\left(1_{2} 2_{2}-1,2_{1}\right)\left(1_{2} 3_{2}+1_{3} 3_{3}\right)
\end{aligned}
$$

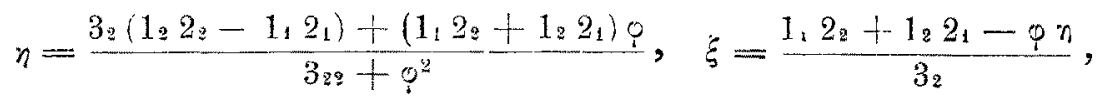

$$
\begin{aligned}
& \theta=\frac{\left(3_{22}+3_{33}\right) n-22\left(1_{2} 3_{2}+1_{3} 3_{3}\right)}{l_{2} 3_{3}-1_{3} 3_{2}}, \quad \zeta=\frac{\left(1_{2} 3_{2}+1_{3} 3_{3}\right) n-22\left(1_{22}+1_{33}\right)}{1_{2} 3_{3}-1_{3} 3_{2}} .
\end{aligned}
$$


8. cas : Les constantes

$$
1_{1}, 1_{2}, 1_{3}, 2_{2}, 2_{3}, 3_{1}, 3_{2}
$$

sont données; la solution de ces cas se construit du cas précédent au moyen de la substitution

$$
\left(1_{1} 2_{1} 3_{1} 1_{3} 2_{3} 3_{3}\right):\left(1_{3} 2_{3} 3_{3} 1_{1} 1_{2} 1_{3}\right) \text {. }
$$

9. ${ }^{\circ}$ cas : Pour exprimer les constantes

$$
\xi=2_{1}, \quad \eta=2_{2}, \quad \zeta=2_{3}, \quad \theta=3_{3}, \quad \varphi=4_{1}
$$

en fonctions des autres coefficients on remarque que les équations

$$
\begin{aligned}
& 1_{1} \xi-1_{2} \eta=3_{1} \varphi+3_{2} 4_{2}, \\
& 1_{2} \xi+1_{1} \eta=3_{2} \dot{\varphi}+3_{1} 4_{2},
\end{aligned}
$$

déterminent $\xi$ et $\eta$ en fonctions linéaires de $\varphi$; ensuite les équations

$$
1_{3} \zeta-4_{1} \theta=1_{1} \xi-3_{1} \varphi, \quad 1_{2} \zeta-4_{2} \theta=1_{3} \eta-3_{2} 4_{3},
$$

donnent $\zeta$ et $\theta$ en fonctions linéaires de $\varphi$; enfin l'équation

$$
\theta \varphi-1_{3} \xi-1_{1} \zeta+3_{1} 4_{3}=0
$$

devient une quadratique pour $\varphi$.

$10 .{ }^{\circ}$ cas : Supposons que

$$
1_{1}, 1_{2}, 1_{3}, 3_{2}, 3_{3}, 4_{1}, 4_{z}
$$

soient des constantes arbitraires; les valeurs des coefficients

$$
\xi=2_{1}, \quad \eta=2_{2}, \quad \zeta=2_{3}, \quad \theta=3_{1}, \quad \varphi=4_{3}
$$

se trouvent de la solution du cas $9 .^{\circ}$ par la substitution

$$
\left(\theta \varphi 3_{1} 3_{2} 4,4_{3}\right):\left(\%, \theta 4,43_{2} 3_{3}\right) \text {. }
$$

11. cas : Il faut qu'on trouve les expressions qui expriment les constantes

$$
\xi=3_{1}, \quad n=3_{2}, \quad \zeta=3_{3}, \quad 0=4_{3}, \quad \varphi=2_{1}
$$

en fonctions des autres paramètres; les formules cherchées se déduisent de la solution du cas $7 .^{\circ}$ au moyen de la substitution

$$
\left(\xi \zeta \theta ; 1_{1} 1_{3} 2_{1} 2_{3} 3_{2} 3_{3} 4_{4} 4\right):\left(\zeta \xi \varphi \theta 1_{3} 1_{1} 2_{3} 2_{1} 4_{2} 4_{1} 3_{3} 3_{2}\right) \text {. }
$$

12. ${ }^{\circ}$ cas: On demande les formes des paramètres

$$
\xi=3_{1}, \quad \eta=3_{2}, \quad \zeta=3_{3}, \quad \theta=4_{1}, \quad \varphi=2_{3}
$$


en fonctions des autres coefficients; la solution se dérive du cas $11 .^{\circ}$ au moyen de la substitution

$$
\left(\xi \zeta 1_{1} 1_{3} 2_{3} 4_{1} 4_{2}\right):\left(\zeta \xi 1_{3} 1_{1} 2_{1} 4_{3} 4_{2}\right) .
$$

$13 .^{\circ}$ cas : Des cinq inconnues

$$
\xi=3_{2}, \quad \eta=3_{3}, \quad \zeta=2_{2}, \quad \theta=2_{3}, \quad \varphi=4_{3},
$$

les paramètres $\xi$ et $\zeta$ sont déterminées par les équations

$$
4_{1} \xi-1_{2} \zeta=3_{1} 4_{1}-1_{1} 2_{1}, \quad 4_{1} \xi-1_{1} \zeta=3_{1} 4_{2}-1_{2} 2_{1} ;
$$

les constantes $\varphi$ et $\eta$ sont données en fonctions linéaires de $\theta$ des équations

$$
\xi \varphi+4_{2} \eta=1_{2} \theta+1_{3} \zeta, \quad 3_{1} \varphi+4_{1} \eta=1_{1} \theta+1_{3} 2_{1}
$$

enfin l'équation

$$
\varphi \eta-4_{2} \xi+1_{2} \zeta-1_{3} \theta=0
$$

est une quadratique pour $\theta$.

$14 .^{\circ}$ cas: Il s'agit d'exprimer les coefficients

$$
\xi=3_{2}, \quad \eta=3_{3}, \quad \zeta=2_{1}, \quad \theta=2_{3}, \quad \varphi=4_{3}
$$

en termes des autres coefficients; la solution est simple: les équations

$$
4_{2} \xi+1_{1} \zeta-1_{2} 2_{2}-3_{1} 4_{1}=0, \quad 4_{1} \xi+1_{2} \zeta+1_{1} 2_{2}-3_{1} 4_{2}=0,
$$

déterminent $\xi$ et $\zeta$; les équations

$$
\xi \varphi+4_{2} n-1_{2} \theta-1_{3} 2_{2}=0, \quad 3_{1} \varphi+4_{1} \eta-1_{1} \theta-1_{3} \zeta=0,
$$

donnent $\varphi$ et $n$ en fonctions de $\theta$; et l'équation

$$
\varphi n-4_{2} \xi-1_{3} \theta+1_{2} 2_{2}=0
$$

devient une quadratique pour 0 .

15. cas: Les paramètres $\xi$ et $\theta$ des cinq paramètres inconnus

$$
\xi=2_{2}, \quad \eta=2_{3}, \quad \zeta=3_{1}, \quad \xi=3_{2}, \quad \varphi=4_{3}
$$

se déterminent en fonctions linéaires de $\zeta$ au moyen des équations

$$
1_{2} \xi-4_{2} \theta=4_{1} \zeta-1_{1} 2_{1}, \quad 1_{1} \zeta-4_{1} \theta=4_{2} \zeta-1_{2} 2_{1} ;
$$

les constantes $n$ et $\varphi$ sont des fonctions linéaires fractionelles des équations

$$
1_{3} n-3_{3} \varphi=1_{1} 2_{1}-4_{1} \zeta, \quad 1_{1} n-\zeta \varphi=3_{1} 4_{1}-1_{3} 2_{1} ;
$$


enfin l'équation

$$
1_{3} \xi+1_{2} \eta-\theta-3_{3} 4_{2}=0
$$

est une quadratique pour $\zeta$.

16. ${ }^{\circ}$ cas: La solution de ce cas, savoir

$$
\xi=i_{2}, \quad \eta=1_{3}, \quad \zeta=3, \quad \theta=3_{3}, \quad \varphi=4_{3},
$$

est une conséquence immédiate du cas $130^{\circ}$ et de la substitution

$$
\left(\xi \eta \zeta 61_{1} 1_{2} 1_{3} 2_{1}\right):\left(\zeta \theta \xi \eta 2_{1} 2_{2} 2_{3} 1_{1}\right) \text {. }
$$

17. ${ }^{\circ}$ cas: On cherche

$$
\xi=3, \quad \eta=3_{3}, \quad \zeta=1_{3}, \quad \xi=1_{1}, \quad \varphi=4_{3}
$$

en fonctions des autres constantes et on trouve $\xi$ et $\theta$ des équations linéaires

$$
4_{2} \xi+2_{1} \theta=1_{2} 2_{2}+3_{1} 4_{1}, \quad 4_{1} \xi-2, \theta=1_{2} 2_{1}-3_{1} 4_{2}
$$

les équations

$$
4_{2} \eta+\xi \varphi=2_{2} \zeta+1_{2} 2_{3}, \quad 4_{1} n+3_{1} \varphi=2_{1} \zeta+2_{3} \theta
$$

deviennent des équations linéaires par rapport aux $\zeta, n, \varphi$ et elles déterminent $n$ et $\varphi$ en fonctions linéaires de $\zeta$; enfin l'équation

$$
\xi \eta+4 \xi-2 \zeta+1_{2} 2_{2}=0
$$

est une quadratique pour $\zeta$.

$18 .^{\circ}$ cas: Les équations

$$
2_{1} \zeta-2_{2} \theta=3_{1} 4_{1}-4_{2} \xi, \quad 2, \zeta+2_{1} \theta=3_{1} 4_{2}+4_{1} \xi,
$$

expriment $\zeta$ et $\theta$ des einq quantités

$$
\xi=3_{2}, \quad \eta=3_{3}, \quad \zeta=1_{1}, \quad \theta=1_{2}, \quad \varphi=4_{3},
$$

en fonctions linéaires de $\xi$; les paramètres $n$ et $\varphi$ sont déterminées en fonctions linéaires fractionnelles de $\xi$ par les équations

$$
4_{2} \eta+\xi \varphi-2_{3} \theta-1_{3} 2_{2}=0, \quad 4_{1} \eta+3 q-2_{3} \zeta-1_{3} z_{1}=0 ;
$$

et $\xi$ est une racine de l'équation quadratique

$$
\varphi \eta+2_{2} \theta-4_{2} \xi-1_{3} 2_{3}=0 \text {. }
$$

19. ${ }^{\circ}$ cas: Les constantes arbitraires sont

$$
\xi=2_{z}, \quad \eta=2_{3}, \quad \zeta=1_{2}, \quad \theta=1_{3}, \quad \varphi=4_{3} ;
$$


$\zeta$ est une fonction linéaire de $\xi$ donnée par l'équation

$$
2_{1} \zeta+1_{1} \xi-3_{1} 4_{2}-3_{2} 4_{1}=0 ;
$$

$\xi$ est une solution de l'équation quadratique

les équations

$$
\xi \zeta-1_{1} 2_{1}+3_{1} 4_{1}-3_{2} 4_{2}=0
$$

$$
1_{1} \eta-3_{1} \varphi=3_{3} 4_{1}-2_{1} \theta, \quad \zeta \eta-3_{2} \varphi=3_{3} 4_{2}-\xi \theta,
$$

déterminent $\eta$ et $\varphi$ en fonctions linéaires de $\sigma$; enfin l'équation

$$
\eta \theta-3_{3} \varphi-1_{1} 2_{1}+3_{1} 4_{1}=0
$$

est une quadratique en $\theta$.

$20 .^{\circ}$ cas: Les constantes

$$
\xi=2_{2}, \quad \eta=2_{3}, \quad \zeta=1_{1}, \quad \theta=1_{3}, \quad \varphi=4_{3}
$$

s'expriment en fonction des sept autres paramètres en observant que l'équation

$$
1_{2} \xi-2 \zeta+34_{1}-3_{2} 4_{2}=0
$$

détermine $\xi$ en fonction linéaire de $\zeta$; que l'équation

$$
\xi \zeta+1_{2} 2_{1}-34_{3}-3_{2} 4_{1}=0
$$

est une quadratique en $\zeta$; que les paramètres $n$ et $\theta$ sont des fonctions linéaires de $\varphi$ des équations

$$
\xi 6+1_{2} \eta=3_{2} \varphi+3_{3} 4_{2}, \quad \zeta \eta+2_{1} \theta=3_{1} \varphi+3_{3} 4_{1},
$$

et enfin que l'équation

$$
\eta \theta-1_{2} \xi-3_{3} \varphi+3_{2} 4_{2}=0
$$

devient une quadratique en $\varphi$.

21. ${ }^{\circ}$ cas: On obtient les formules pour les valeurs de

$$
\xi=2_{2}, \quad \eta=2_{3}, \quad \zeta=1_{1}, \quad G=1_{2}, \quad \varphi=4_{3}
$$

de la manière suivante: l'équation

$$
2_{1} \zeta-\xi \theta-3_{1} 4_{1}+3_{2} 4_{2}=0
$$

donne $\zeta$ en fonction linéaire de $\xi 6$; de l'équation

$$
\xi \zeta+2_{1} \theta-3_{1} 4_{2}-3_{2} 4_{1}=0
$$


on voit que $\theta$ est une fonction dont le numérateur est une fonction linéaire, et le dénominateur une fonction quadratique, de $\xi$; maintenant on remarque que $\zeta$ devient une fonction de $\xi$ de la même forme que la fonction $\theta$; en substituant ces valeurs de $\zeta$ et $\theta$ dans les équations

$$
1_{3} \eta-3_{3} \varphi-\xi \theta+3_{z} 4_{2}=0, \quad \theta \eta-3_{2} \varphi+1_{3} \xi-3_{3} 4_{2}=0
$$

on trouve pour $\eta$ une cubique fonction de $\xi$ sur une fonction quadratique de $\xi$, et pour. est une racine de l'équation du einquième degré

$$
\eta \zeta-3_{1} \varphi+1_{3} 2_{1}-3_{3} 4_{1}=0 .
$$

22. ${ }^{\circ}$ cas : Des paramètres

$$
\xi=3_{1}, \quad \eta=3_{2}, \quad \zeta=4_{1}, \quad \theta=4_{2}, \quad \varphi=2_{i},
$$

$\zeta$ est une fonction linéaire de $\xi$ donnée par l'équation

$$
3_{3} \zeta+4_{3} \xi-1_{3} 2_{1}-1_{1} 2_{3}=0 ;
$$

$\xi$ est une racine de l'équation quadratique

$$
\xi \zeta+1_{3} 2_{3}-1_{1} 2_{1}-3_{3} 4_{3}=0 ;
$$

$\eta$ et $\theta$ sont des fonctions linéaires de $\varphi$ données par les áquations

$$
\zeta \eta-1_{1} q+\xi \theta-1_{2} 2_{1}=0, \quad 4_{3} \eta-1_{3} \varphi+3_{3} \theta-1_{2} 2_{3}=0 ;
$$

et $\varphi$ est une racine de la quadratique

$$
n \theta-1_{2} i+1_{3} 2_{3}-3_{3} 4_{3}=0 \text {. }
$$

$23 .^{\circ}$ cas: Les systèmes des équations pour déterminer les paramètres

$$
\xi=3_{1}, \quad \eta=3_{2}, \quad \zeta=2_{1}, \quad \theta=2_{z}, \quad \varphi=4_{i},
$$

est linéaire; $\zeta$ est égale

$\xi$ est donnée de l'équation

$$
\frac{34_{1}-34_{3}+1_{3} 2_{3}}{1_{1}}
$$

$$
4_{3} \xi=1_{3} \zeta+1_{1} 2_{3}-3_{3} 4_{1}
$$

enfin, $n, \theta, \varphi$ sont des solutions du système linéaire

$$
\begin{gathered}
1_{1} \theta-3_{1} \varphi-4_{1} \eta+1_{2} \zeta=0, \quad 1_{2} 9-3_{2} \varphi-1_{3} 2_{3}+3_{3} 4_{3}=0 \\
1_{3} \theta-3_{3} \varphi-4_{3} \eta+1_{2} 2_{3}=0 .
\end{gathered}
$$


24. ${ }^{\circ}$ cas: On détermine les paramètres

$$
\xi=4_{1}, \quad \eta=4_{2}, \quad \zeta=2_{1}, \quad \theta=2_{2}, \quad \varphi=3_{2}
$$

de la manière suivante: $\xi$ et $\zeta$ sont solutions des équations

$$
3_{1} \xi-1_{1} \zeta=3_{3} 4_{3}-1_{3} 2_{3}, \quad 3_{3} \xi-1_{3} \zeta=1_{1} 2_{3}-34_{3} ;
$$

$\varphi$ et $\eta$ sont fonctions linéaires de $\theta$ données par les équations

$$
1_{1} \theta+1_{2} \zeta-3_{1} n-\varphi \xi=0, \quad 1_{3} \theta-4_{3} \varphi-3_{3} n+1_{2} 2_{3}=0 ;
$$

et $\theta$ est une racine de la quadratique

$$
\varphi \eta-1_{2} \theta+1_{3} 2_{3}-3_{3} 4_{3}=0 \text {. }
$$

25. ${ }^{\circ}$ cas: Il s'agit d'exprimer

$$
\xi=2_{1}, \quad \eta=2_{2}, \quad \zeta=3_{1}, \quad \theta=3_{2}, \quad \varphi=4_{3},
$$

en fonction des autres paramètres; les équations

$$
1_{1} \xi-4_{1} \zeta-1_{2} \eta+4_{2} \theta=0, \quad 1_{2} \xi+1_{1} \eta-4 \zeta-4_{1} \theta=0
$$

déterminent $\xi$ et $\zeta$ en fonctions linéaires de $\eta$ et $\theta$; on substitue ces valeurs de $\xi$ et $\zeta$ dans l'équation

$$
\varphi \zeta-1_{3} \xi-1_{1} 2_{3}+3_{3} 4_{1}=0,
$$

et on élimine $\varphi \theta$ au moyen de l'équation

$$
\theta-1_{3} \eta-1_{2} 2_{3}+3_{3} 4_{2}=0
$$

l'équation résultante et l'équation

$$
1_{2} \eta-4_{2} \theta+33_{3} \varphi-1_{3} 2_{3}=0
$$

donnent $\varphi$ et $\theta$ en fonction de $\eta$; enfin l'équation

$$
\varphi \theta-1_{3} \eta-1_{2} 2_{3}+3_{3} 4_{2}=0
$$

devient une quadratique en $\eta$.

$26 .^{\circ}$ cas : Les formes des paramètres

$$
\xi=1_{1}, \quad \eta=1_{2}, \quad \zeta=3_{1}, \quad \theta=3_{2}, \quad \varphi=4_{3}
$$

en fonction des autres constantes se déduisent du cas $25 .^{\circ}$ par la substitution

$$
\left(1,1 \quad 1_{3} 2_{3}\right):\left(2,2,2,1_{3}\right)
$$


$27^{\circ}$ cas : Pour obtenir les paramètres

$$
\xi=1_{1}, \quad \eta=1_{2}, \quad \zeta=2_{1}, \quad \theta=2_{2}, \quad \varphi=4_{3},
$$

il est nécessaire de résoudre le système des équations
(i) $\xi \zeta-\eta \theta-3_{1} 4_{1}+3_{z} 4_{2}=0$,
(ii) $n \theta+3_{3} \varphi--1_{3} 2_{3}-3_{2} 4_{2}=0$,
(iii) $\xi \theta+n \zeta-3_{1} 4_{2}-3_{2} 4_{1}=0$,
(iv) $2_{3} n+1_{3} \theta-3_{2} \varphi-3_{3} 4_{2}=0$,

(v) $2_{3} \xi+1_{3} \zeta-33_{1} \varphi-3_{3} 4_{1}=0$;

des équations (ii) et (iv) on a

$$
\eta \theta+a \eta+b \theta-c=0
$$

des équations (i), (ii) et (iv) on trouve

$$
\xi \zeta+e \xi+f \zeta-k=0
$$

pour l'équation (iii) on peut écrire

$$
\xi \theta+\eta \zeta-l=0
$$

les équations (iv) et (v) donnent

$$
\lambda \xi+\mu \eta+\nu \zeta+\rho \theta+\sigma=0
$$

de ce système il résulte que $\eta$ et $\zeta$ sont déterminées par les équations quartiques

$$
\begin{aligned}
& (a n-c)(f \zeta-k)+(\eta \zeta-l)(\eta+b)(\zeta+e)=0, \\
& \lambda(f \zeta-k)(\eta+b)+\rho(a n-c)(\zeta+e)-(\mu \eta+\nu \zeta+\sigma)(\eta+b)(\zeta+e)=0 .
\end{aligned}
$$

28..$^{\circ}$ cas: Les équations pour déterminer

$$
\xi=2_{1}, \quad \eta=2_{3}, \quad \zeta=1_{3}, \quad \theta=3_{3}, \quad \varphi=4_{3}
$$

demandent que

$$
\xi=\frac{1_{2} 2_{2}-3_{2} 4_{2}+3_{1} 4_{1}}{1_{1}}=\frac{34_{2}+324_{1}-1_{1} 2_{2}}{1_{2}} ;
$$

donc toutes les sept paramètres

$$
1_{1}, 1_{2}, 2_{2}, 3_{1}, 3_{2}, 4_{1}, 4_{2}
$$

ne peuvent pas être arbitraires.

$29 .^{\circ}$ cas: Les formes des paramètres

$$
\xi=2_{1}, \quad \eta=2_{2}, \quad \zeta=1_{3}, \quad \theta=3_{1}, \quad \varphi=4_{3}
$$


on trouve de la manière suivante: le système à résoudre est

$$
\begin{gathered}
a \xi+b \eta+c \theta-k=0, \quad b \xi+a n+h o-l=0, \quad f \zeta+g \varphi+e n-k=0, \\
\eta \zeta+j \varphi-m=0, \quad \xi \zeta-\varphi \theta-n=0
\end{gathered}
$$

la cinquième équation devient la quadratique

$$
\begin{aligned}
& \left.\mid l j(h b-a c)+e(b k-a l)+k\left(b^{2}-a^{2}\right)\right\} \eta^{2} \\
& +\left\{(a c-b h)(j k-g m)-e j(h k-c l)+f m\left(b^{2}-a^{2}\right)-\right. \\
& -k(a l-b k)+g n(a h-b c) \mid \eta \\
& +(h k-c)(j k-g m)-f m(a l-b k)-n j(a h-b c)=0
\end{aligned}
$$

en substituant les fonctions $\xi, \theta$, $\zeta$, et $\rho$ de $n$ déduites des autres équations. 30. ${ }^{\circ}$ cas: Des cinq paramètres

$$
\xi=2_{2}, \quad \eta=2_{3}, \quad \zeta=1_{3}, \quad \theta=3_{2}, \quad \rho=4_{3},
$$

$\xi$ et $\theta$ se déterminent par les équations

$$
1_{2} \xi-4_{2} \theta-1_{1} 2_{1}+3_{1} 4_{1}=0, \quad 1_{1} \xi-4_{1} \theta+1_{2} 2_{1}-3_{1} 4_{2}=0 ;
$$

et les trois équations

$$
\begin{gathered}
\xi \zeta-\varphi \theta+1_{2} \eta-3_{3} 4_{2}=0, \quad 1_{1} \eta+2, \zeta-3_{1} \varphi-3_{3} 4_{1}=0 \\
\zeta \eta-1_{2} \xi+4_{2} \theta-3_{3} \varphi=0
\end{gathered}
$$

donnent une quadratique pour $\varphi$.

$31 .^{\circ}$ cas: La solution de ce cas, c'est-à-dire la détermination des formes de

$$
\xi=2_{1}, \quad n=2_{3}, \quad \zeta=1_{3}, \quad \theta=3_{3}, \quad \varphi=4_{1},
$$

suivit immédiatement du cas précédent au moyen de la substitution

$$
\left(\theta \varphi 1_{1} 1_{2} 2,3_{1} 3_{3} 4_{1} 4_{2}\right):\left(\varphi \theta 1_{2} 1_{1} 2_{2} 4_{2} 4_{3} 3_{2} 3_{1}\right)
$$

32. cas: Le système des équations pour trouver

$$
\xi=2_{1}, \quad \eta=2_{2}, \quad \zeta=1_{3}, \quad \theta=3_{3}, \quad \varphi=4_{2}
$$

en fonction des autres paramètres est

$$
\begin{gathered}
1_{1} \xi-1_{2} \eta+3_{2} \varphi-3_{1} 4_{1}=0, \quad 1_{2} \xi+1_{1} \eta-3_{1} \varphi-34_{1}=0, \\
1_{2} \eta-2_{3} \zeta+4_{3} \theta-3_{2} \varphi=0, \\
\eta \zeta-\theta \varphi+1_{2} 2_{3}-3_{2} 4_{3}=0, \quad \xi \zeta-4_{1} \theta+1_{1} 2_{3}-3_{1} 4_{3}=0 ;
\end{gathered}
$$


on voit sans difficulté qu'on a

$$
\eta=L_{1}(\xi), \quad \varphi=L_{2}(\xi), \quad \zeta=\frac{L_{3}(\xi)}{L_{1}(\xi)}, \quad \theta=\frac{Q_{1}(\xi)}{L_{1}(\xi)},
$$

où $L$ est une abbreviation de fonction linéaire et $Q$ est une abbreviation de fonction quadratique; enfin $\xi$ est déterminée par l'équation cubique:

$$
L_{1}(\xi) L_{3}(\xi)-L_{2}(\xi) Q_{1}(\xi)-c L_{1}(\xi)=0 .
$$

33. ${ }^{\circ}$ cas: Supposons que les constantes soient

les équations

$$
\xi=2, \quad \eta=2, \quad \zeta=1_{3}, \quad \theta=3_{1}, \quad \varphi=4_{1} ;
$$

$$
\zeta \eta+1_{2} 2_{3}-3_{2} 4_{3}-3_{3} 4_{3}=0, \quad 2_{3} \zeta-1_{2} \eta+3_{2} 4_{2}-3_{3} 4_{3}=0,
$$

donnent deux valours à chacune des paramètres $\zeta$ et $n$; les quantités $\theta$ et $\varphi$ s'expriment en fonction de $\xi$ par les équations

$$
1_{1} \eta+1_{2} \xi-4_{2} \theta-3_{2} \varphi=0, \quad \xi \zeta-4_{3} \theta-3_{3} \varphi+1_{2} 2_{3}=0
$$

et l'équation

$$
\varphi \theta-1_{1} \xi+1_{2} n-3_{2} 4_{2}=0
$$

est une quadratique en $\xi$.

$34 .^{\circ}$ cas: Quelles fonctions des autres paramètres doivent être les constantes

$$
\xi=2_{\imath}, \quad \eta=2_{3}, \quad \zeta=1_{3}, \quad \theta=3_{i} ; \quad \varphi=4_{1} \text { ? }
$$

Des équations

$$
1_{2} \xi-4, \theta+3_{1} \varphi-1_{1} 2_{1}=0, \quad 1_{1} \xi-\varphi \theta+1_{2} 2_{1}-3_{1} 4_{2}=0,
$$

on écrit les formes suivantes de $\xi$ et $\theta$ :

des équations

$$
\xi=Q_{1}(\varphi)\left|L_{z}(\varphi), \quad \theta=L_{1}(\varphi)\right| L_{2}(\varphi)
$$

$$
\xi \zeta+1_{2} n-4_{3} \theta-3_{3} 4_{2}=0, \quad 1_{1} n+2_{1} \zeta-3_{3} \varphi-3_{1} 4_{3}=0,
$$

on a

enfin l'équation

$$
n=C_{1}(\varphi)\left|Q_{3}(\varphi), \quad \zeta=Q_{2}(\varphi)\right| Q_{3}(\varphi)
$$

est une sextique

$$
\zeta \eta-1_{2} \xi+4_{2} \theta-3_{3} 4_{3}=0
$$

$$
\begin{gathered}
L_{2}(\varphi) Q_{2}(\varphi) C_{1}(\varphi)-1_{2} Q_{1}(\zeta) Q_{3}^{\prime}(\varphi)+4_{3} L_{1}(\varphi) Q_{3}(\varphi)- \\
-3_{3} 4_{3} L_{2}(\varphi) Q_{3}^{2}(\varphi)=0 .
\end{gathered}
$$


$35 .^{\circ}$ cas: Pour trouver les valeurs des constantes

$$
\xi=2_{1}, \quad n=2_{2}, \quad \zeta=1_{3}, \quad \theta=3_{1}, \quad \varphi=4_{2}
$$

on remarque que les équations

donnent

$$
4_{1} \theta+2_{3} \zeta=1_{1} \xi+3_{3} 4_{3}, \quad 4_{3} \theta-\xi \zeta=1_{1} 2_{3}-3_{3} 4_{1},
$$

et les équations

$$
\theta=Q_{1}(\xi)\left|L_{2}(\xi), \quad \zeta=L_{1}(\xi)\right| L_{2}(\xi)
$$

donnent

$$
3_{2} \varphi-1_{2} \eta=4_{1} \theta-1_{1} \xi, \quad 6 \varphi-1_{1} \eta=1_{2} \xi-3_{2} 4_{1},
$$

enfin l'équation

$$
\varphi=Q_{2}(\xi) \mid Q_{3}(\xi), \quad n=\frac{\text { Quartique }(\xi)}{L_{2}(\xi) Q_{3}(\xi)}
$$

devient la quintique

$$
\zeta \eta-3_{3} \varphi+1_{2} 2_{3}-3_{2} 4_{3}=0
$$

Quartique $(\xi) L_{1}(\xi)+\left(1_{2} 2_{3}-3_{3} 4_{3}\right) L_{2}^{2}(\xi) Q_{3}(\xi)-3_{3} L_{2}^{2}(\xi) Q_{2}(\xi)=0$

en $\xi$.

$36 .^{\circ}$ cas: Les constantes à trouver dans ce cas sont

$$
\xi=2_{1}, \quad \eta=2_{2}, \quad \zeta=1_{3}, \quad \theta=3_{2}, \quad \varphi=4_{2} ;
$$

les paramètres $\xi$ et $\zeta$ se déterminent des équations

$$
1_{1} \xi-2_{3} \zeta=3_{1} 4_{1}-3_{3} 4_{3}, \quad \xi \zeta=3_{3} 4_{1}+34_{3}-1_{1} 2_{3}
$$

$\eta$ et $\theta$ sont des fonctions linéaires de $\varphi$ des équations

$$
1_{2} \xi+1_{1} \eta-4_{1} \theta-3_{1} \varphi=0, \quad 1_{3} \xi-4_{3} \theta-3_{3} \varphi+1_{2} 2_{3}=0 ;
$$

et l'équation

$$
\theta p-1_{2} n+2 \zeta-2_{3} 4_{3}=0
$$

devient une quadratique en $\varphi$.

37. ${ }^{\circ}$ cas: Il s'agit de déterminer les formes des paramètres

$$
\xi=2_{1}, \quad \eta=2_{2}, \quad \zeta=1_{3}, \quad \theta=3_{3}, \quad \varphi=4_{3} ;
$$

en formant les équations de condition on trouve $\xi$ et $\eta$ immédiatement; $\theta$ et $\varphi$ sont des fonctions linéaires de $\zeta$ qui se détermine par la résolution d'une quadratique.

$38^{\circ}$ cas: Les inconnues

$$
\xi=2_{2}, \quad \eta=2_{3}, \quad \zeta=1_{3}, \quad \theta=3_{1}, \quad \varphi=4_{3} ;
$$


on construit $\theta$ et $\varphi$ immédiatement; $\zeta$ et $\eta$ sont des fonctions linéaires de $\varphi$ qui est une racine d'une quadratique.

$39 .^{\circ}$ cas: Supposons que les constantes à déterminer sont

$$
\xi=2, \quad \eta=2_{3}, \quad \zeta=1_{3}, \quad 6=3_{2}, \quad \varphi=4_{3}
$$

on trouve $\xi$ et $\theta$ sans difficulté; $\eta$ et $\zeta$ sont linéaire en $\varphi$, et $\varphi$ est donnée par une équation quadratique

40. ${ }^{\circ}$ cas: Des quantités

$$
\xi=2_{3}, \quad n=2_{3}, \quad \zeta=1_{3}, \quad \theta=3_{3}, \quad \varphi=4_{1},
$$

on trouve $\xi$ et $\xi$; les paramètres $\eta$ et $\zeta$ sont des fonctions linéaires de $\theta$, et $\theta$ est une solution d'une quadratique.

41. ${ }^{\circ}$ cas: On détermine les formes des paramètres

$$
\xi=2_{1}, \quad \eta=2_{3}, \quad \zeta=1_{3}, \quad \theta=3_{3}, \quad \varphi=4_{2}
$$

au moyen de la résolution du cas $39 .^{\circ}$ et la substitution

$$
\left(\theta \varphi 3,3_{3} 4_{1} 4_{2}\right):\left(\varphi 64,4_{3} 3_{1} 3_{2}\right) \text {. }
$$

42. ${ }^{\circ}$ cas : La construction des formes des constantes

$$
\xi=2_{2}, \quad \eta=2_{3}, \quad \zeta=1_{3}, \quad \theta=3_{1}, \quad \varphi=4_{1}
$$

n'est pas si simple; des équations

$$
1_{2} \xi-\eta \zeta=3_{2} 4_{2}-3_{3} 4_{3}, \quad \xi \zeta+1_{2} \eta=3_{2} 4_{3}+3_{3} 4_{2},
$$

nous avons

les équations

$$
\xi=L_{1}(\zeta)\left|Q_{1}(\zeta), \quad n=L_{2}(\zeta)\right| Q_{1}(\zeta)
$$

$$
1_{1} \xi-4_{2} \theta-3_{2} \varphi+1_{2} 2_{1}=0, \quad 1_{1} \eta+2_{1} \zeta-4_{3} \theta-3_{3} \varphi=0
$$

donnent

$$
\theta=\frac{C_{2}(\zeta)}{\left(324_{3}-34_{2}\right) Q_{1}(\zeta)}, \quad q=\frac{C_{1}(\zeta)}{\left(324_{3}-344_{2}\right) Q_{1}(\zeta)}
$$

enfin l'équation

devient la sextique

$$
\theta \varphi+1_{2} \xi-1_{1} 2_{1}-3_{z} 4_{2}=0
$$

$C_{1}(\zeta) C_{2}(\zeta)+\left(3_{2} 4_{3}-3_{3} 4_{2}\right)\left\{1_{2} L_{1}(\zeta) Q_{1}(\zeta)-\left(1_{1} 2_{1}+3_{2} 4_{2}\right) Q_{1}^{2}(\zeta)\right\}=0$.

43. ${ }^{\circ}$ cas : La solution de ce cas, savoir

$$
\xi=2, \quad n=2_{3}, \quad \zeta=1_{s}, \quad \theta=3, \quad \varphi=4_{1}
$$


se déduit du cas $34 .^{\circ}$ par la substitution

$$
\left(\theta \varphi 1_{1} 1_{2} 2_{1} 3_{3} 3_{1} 4_{2} 4_{3}\right):\left(\varphi \theta 1_{2} 1_{1} 2_{2} 4_{3} 4_{2} 3_{1} 3_{3}\right)
$$

$44 .^{\circ}$ cas : Ce cas

$$
\xi=2_{2}, \quad \eta=2_{3}, \quad \zeta=1_{3}, \quad \theta=3_{1}, \quad \varphi=4_{2},
$$

est une conséquence directe du cas $34 .^{\circ}$ et de la substitution

$$
\left(6 \varphi 3_{3} 3_{1} 4_{2} 4_{3}\right):\left(\varphi \theta 4_{3} 4_{1} 3_{2} 3_{3}\right) \text {. }
$$

$45 .^{\circ}$ cas: Les formes des constantes

$$
\xi=2_{1}, \quad \eta=2_{3}, \quad \zeta=1_{3}, \quad \theta=3_{2}, \quad \varphi=4_{2},
$$

se déterminent de la solution du cas $42 .^{\circ}$ au moyen de la substitution

$$
\left(1,1_{2} 2,3,4\right):(1,1,2,3,4) \text {. }
$$

En tentant de trouver de semblables transformations dans les espaces à un plus grand nombre de dimensions, on peut chercher à déterminer les formes des fonctions qui définissent la correspondance voulue en exprimant les conditions que le déterminant correspondant sera capable de représenter le premier membre de l'équation d'une hypersphère.

Ainsi, si dans l'espace à $n$ dimensions, la ligne droite

$$
\sum_{i}^{n} \alpha_{i, j} x_{j}+\alpha_{i, 0}=0, \quad i=1,2, \ldots, n-1
$$

doit être transformée en sphère par la correspondance établie par les équations

$$
\begin{aligned}
& \sum_{1}^{n} \varphi_{i}\left(X_{1}, X_{2}, \ldots, X_{n}\right) x_{i}+\varphi_{0}\left(X_{1}, X_{2}, \ldots, X_{n}\right)=0 \\
& \sum_{i}^{n} \psi_{i}\left(X_{1}, X_{2}, \ldots, X_{n}\right) x_{i}+\psi_{0}\left(X_{1}, X_{2}, \ldots, X_{n}\right)=0,
\end{aligned}
$$

l'équation

$$
\left|\alpha_{1,0}, \alpha_{2,1}, \ldots, \varphi_{n-1}, \psi_{n}\right|=0
$$

doit se réduire à la forme

$$
\sum_{i}^{n} X_{i}\left(X_{i}+\beta_{i}\right)+\gamma=0, \quad \beta_{i}, \gamma \text { constantes }
$$

mais cette réduction est en général impossible, ce qui est clajr et de la géométrie et de la théorie des formes algébriques. 
Cependant pour l'espace à quatre dimension on trouve que la transformation curieuse déterminée par les équations directrices

$$
\begin{aligned}
& x+(Z-i W) z-(X+i Y)(w+1)=0, \\
& y-(X-i Y) z-(Z+i W)(w+1)=0
\end{aligned}
$$

transforme actuellement les droites arbitraires en sphères.

Les propriétés caractéristiques de cette transformation-ci se révèlant sans peine en notant que la droite

$$
w+l z+a=0, \quad x+m z+b=0, \quad y+n z+c=0
$$

est transformée en la sphère

$$
\left|\begin{array}{ccc}
l & 1 & a-1 \\
Z-i W-m & -(X+i Y) & -b \\
X-i Y+n & Z+i W & c
\end{array}\right|=0 .
$$

Parmi ces propriétés on peut remarquer : $10^{\circ}$ que la transformation n'est pas une trasformations de contact, comme le criterinm de LIE le montre; $2 .^{\circ}$ qu'elle dégénère en le transformations de Lje quand la quatrième dimension s'annule; $3 .^{\circ}$ que la correspondance directe est $(1,1)$, mais que l'inverse est $\left(1, \infty^{1}\right) ; 4 .^{\circ}$ que si une droite réelle doit être transformée en une sphère la sphère se réduit à un point, mais qu'autrement le rayon de la sphère est différent de zéro.

Il n'échappera pas à l'observation du lecteur que la méthode dont on s'est servi ci-dessus peut être employée à construire des correspondances entre les lignes, les plans et les hyperplans des ordres divers d'un espace à $n$ dimensions, et les hypersurfaces de cet espace, respectivement, non seulement dans le cas de deux équations directrices de la forme ci-dessus, mais aussi dans le cas de $\nu$ telles équations de cette forme, où $\nu$ peut avoir une valeur quelconque entre 1 et $n$; en particulier le cas où $\nu=n$, quand il est possible, donne des transformations ponctuelles. Enfin on voit que ces correspondances forment des léaisons entre les géométries des hyperplans des ordres divers et la géométrie des hypersphères tout-à-fait analogues à la liaison entre la géométrie de droites et la géométrie des sphères de l'espace ordinaire. 


\section{LES TRANSFORMATIONS DE CONTACT ENTRE LES SPHלेReS DE L'ESPACE A $n$ DIMENSIONS.}

Si on désigne par $\xi_{1}, \xi_{2}, \ldots, \xi_{n}$ les coordonnées du centre, et par $\rho$ le radius de la sphère, il faut que l'équation

$$
\sum_{i}^{n} d \xi_{i}^{2}-d \rho^{2}=0
$$

soit une équation invariante sous toutes les transformations cherchées, car, de la définition d'une transformation de contact sphères tangentes doivent être changées en sphères tangentes; donc, ce qui on sait bien, le problème se référe à la détermination des transformations conformes ponctuelles de l'espace $\left(\xi_{1}, \xi_{2}, \ldots, \xi_{n}, \rho \sqrt{-1}\right)$ à $n+1$ dimensions; la solution de ce pro. blème-ci Lie a trouvé (LIE-Enger, Theorie der Transformationsgruppen, tome III), savoir que toutes ces transformations conformes constituent un groupe à $\frac{1}{2}(n+1)(n+2)$ paramètres, engendré par les transformations infinitésimales

$$
p_{j}, \quad x_{j} p_{k}-x_{k} p_{j}, \quad \sum_{1}^{n} x_{l} p_{l}, \quad 2 x_{j} \sum_{l}^{n} x_{l} p_{l}-p_{j} \sum_{1}^{n} x_{l}^{2}, \quad j, k=1,2, \ldots, n .
$$

De la même manière on traite le problème de trouver les transformations ponctuelles entre les variétés applicables de l'espace à $n$ dimensions; M. Staeckel a donné la solution de ce problème dans l'espace ordinaire (Comptes Rendus, 1895); son raisonnement se traduit pour le cas de l'espace à $n$ dimensions par un seul changement des indices.

Soient

$$
V_{1}\left(x_{11}, x_{12}, \ldots, x_{1 n}\right), \quad V_{2}\left(x_{21}, x_{22}, \ldots, x_{2 n}\right)
$$

deux variétés applicables. On se propose de déterminer toutes les transformations

$$
\begin{aligned}
& X_{1 i}=\varphi_{1 i}\left(x_{11}, x_{12}, \ldots, x_{1 n}, x_{21}, x_{22}, \ldots, x_{2 n}\right), \\
& X_{2 i}=\varphi_{i i}\left(x_{11}, x_{12}, \ldots, x_{1 n}, x_{21}, x_{22}, \ldots, x_{2 n}\right),
\end{aligned}
$$


qui changent la couple précédente en une couple de variétés

$$
V_{1}\left(X_{11}, X_{12}, \ldots, X_{1 n}\right), \quad V_{2}\left(X_{21}, X_{22}, \ldots, X_{2 n}\right)
$$

encore applicables.

On fait le changement de variables

$$
x_{2 j}=i x_{2 j}^{\prime}, \quad X_{2 j}=i \cdot X_{2 j}^{\prime}, \quad i=\sqrt{-1}, \quad j=1,2, \ldots, n ;
$$

donc il faut ed il suffit que l'équation

$$
\sum_{1}^{n} j\left(d x_{1 j}^{2}+d x_{2 j}^{\prime \prime \prime}\right)=0
$$

soit une équation invariante sous les transformations

$$
\begin{aligned}
& X_{1 j}=\varphi_{1 j}\left(x_{11}, x_{12}, \ldots, x_{1 n}, i x_{21}^{\prime}, i x_{22}^{\prime}, \ldots, i x_{2 n}^{\prime}\right), \\
& X_{2 j}=\varphi_{2 j}\left(x_{11}, x_{12}, \ldots, x_{1 n}, i x_{21}^{\prime}, i x_{22}^{\prime}, \ldots, i x_{2 n}^{\prime}\right) ;
\end{aligned}
$$

e'est-à-dire que ces équations ci définissent une représentation conforme de la variété $\left(x_{11}, x_{12}, \ldots, x_{1 n}, x_{21}^{\prime}, x_{22}^{\prime}, \ldots, x_{2 n}^{\prime}\right)$ sur la variété $\left(X_{11}, X_{12}, \ldots\right.$, $X_{1 n}, X_{21}^{\prime}, X_{22}^{\prime}, \ldots, X_{2 n}^{\prime}$ ); mais d'après le théorème de LiE toutes ces représentations conformes constituent un groupe de transformations semblable au groupe à $\frac{1}{2}(2 n+1)(2 n+2)$ paramètres de toutes les transformations par rayons vectores réciproques de l'espace à $2 n$ dimensions.

$\dot{A}$ une couple réelle de surfaces $S_{1}, S_{2}$ ils correspondent $\infty^{\frac{1}{2}(2 n+1)(2 n+2)}$ couples réelles $\Sigma_{1}, \Sigma_{2}$; si on exclude les couples qui on peut faire coincider par mouvements et par transformations de similitude, on a $\infty^{n(n+2)}$ différentes couples correspondantes.

\section{LES TRANSFORMATIONS DE CONTACT ENTRE LES LIGNES ASYMPTOTIQURS} ET LES LIGNES DE COURBURE.

Il y a une autre catégorie intéressante de problèmes dans la géométrie des transformations de contact; cette catégorie se compose des questions où on cherche à trouver les transformations qui changent une surface en une surface de la manière que, à une famille des lignes remarquables sur l'une 
surface corresponde une famille remarquable des lignes sur l'autre surface; par exemple, les transformations de contact entre les lignes asymptotiques, les lignes de courbure, les $D$-lignes, les cercles géodésiques, et cetera. Toutes les transformations de contact qui transforment les lignes asymptotiques en lignes asymptotiques sont bien connues; Lie a trouvé par la géométrie que la famille consiste de transformations projectives et dualistiques. Une conséquence la plus importante de la transformation de Liw entre les lignes droites et les sphères est que ses formules font correspondre aux lignes asymtotiques lignes de courbure. LIE a remarqué aussi que toutes les transformations de contact qui transforment les lignes asymptotiques en lignes de courbure font correspondre sphères aux lignes droites. (Voire Mathematische Annalen, tome V; LIE-EvaEc, Theorie der Transformationsgruppen, tome II.) Lis s'est proposé de déterminer toutes les transformations de contact qui conservent les lignes de courbure; et $M$. Darboux donne le résultat suivant, qui résume les recherches de LiE: Les transformations de contact les plus générales qui conservent les lignes de courbure sont celles qui soumettent les six coordonnées homogènes d'une sphère à la transformation linéaire la plus générale qui conserve la relation quadratique entre les coordonnées. (Leçons sur les systemes orthogonaux et les coordonnées curvilignes, tome I, p. 63.) On se propose ici de fairo la détermination analytique de toutes les transformations des deux classes de transformations de contact: $1 .^{\circ}$ les transformations qui changent les lignes asymptotiques en lignes de courbure; $2 .^{\circ}$ les transformations qui conservent les lignes asymptotiques.

Les TRANGFORMATIONS DE CONTACT QUI TRANGFORMENT LES LIGNES ASTMPTOTIQUES EN LIGNES DE COURBURE.

Soit

$$
\left.\begin{array}{c}
X=X(x, y, z, p, q), \quad Y=Y(x, y, z, p, q), \quad Z=Z(x, y, z, p, q) \\
P=P(x, y, z, p, q), \quad Q=Q(x, y, z, p, q)
\end{array}\right\}
$$

la transformation la plus générale cherchée.

On a les relations établies par les théorèmes de LIE, Darboux et 


\section{MAYER}

où

$$
\begin{aligned}
& {[P Q]=[P Y]=[Q X]=[X Y]=[X Z]=[Y Z]=0,} \\
& {\left[\begin{array}{ll}
P X & X
\end{array}=[Q Y]=\frac{1}{P}[P Z]=\frac{1}{Q}[Q Z]=\rho=0, \quad\right\}}
\end{aligned}
$$

ù

$$
\left.\begin{array}{c}
{[\Phi \Psi]==\Phi_{p} \Psi x-\Phi x \Psi_{p}+\Phi_{q} \Psi y-\Phi y \Psi_{q},} \\
\Phi_{p}==\frac{\partial \Phi}{\partial p}, \quad \Phi_{q}=\frac{\partial \Phi}{\partial q}, \quad \Phi^{x}=\Phi_{x}+p \Phi_{z}, \quad \Phi^{y}=\Phi_{y}+q \Phi_{z} .
\end{array}\right\}
$$

Pour que les lignes asymptotiques se transforment en lignes de courbure il faut que la forme

$$
\begin{gathered}
\Omega \equiv\left\{\left(1+P^{2}\right) S-P Q R\right\} d X^{2}+ \\
+\left(\left(1+P^{2}\right) T-\left(1+Q^{2}\right) R\left\{d X d Y+\left\{P Q T-\left(1+Q^{3}\right) S\right\} d Y^{2}\right\}\right.
\end{gathered}
$$

soit égale à zéro en conséquence de l'équation

$$
r d x^{2}+2 s d x d y+t d y^{2}=0 \text {. }
$$

Pour développer la forme (4) on observe d'abord qu'on a (Voire la note de M. Vivantr, Rendiconti Circolo Matematico di Palermo, tome V.)

où

$$
R=\frac{\varphi(P, Y)}{\varphi(X, Y)}, \quad S=\frac{\varphi(X, P)}{\varphi(X, Y)}=\frac{\varphi(Q, Y)}{\varphi(X, Y)}, \quad T=\frac{\varphi(X, Q)}{\varphi(X, Y)},
$$

$$
\begin{gathered}
\varphi(U, V)=U^{(x)} V^{(y)}-U^{(y)} V^{(x)} \equiv \\
\equiv\left|\begin{array}{cc}
U^{x} & V^{x} \\
U^{y} & V_{y}
\end{array}\right|+\left|\begin{array}{cc}
U_{p} & V_{p} \\
U^{y} & V^{y}
\end{array}\right| r+\left\{\begin{array}{cc}
U^{x} & V^{x} \\
U_{p} & V_{p}
\end{array}|+| \begin{array}{cc}
U_{q} & V_{q} \\
U^{y} & V^{y}
\end{array}\right) s+ \\
+\left|\begin{array}{cc}
U^{x} & V^{x} \\
U_{q} & V_{q}
\end{array}\right| t+\left|\begin{array}{cc}
U_{p} & V_{p} \\
U_{q} & V_{q}
\end{array}\right|\left(r t-s^{z}\right), \\
U^{(x)}=U^{x}+r U_{p}+s U_{q}, \quad U^{(y)}=U y+s U_{p}+t U_{q}
\end{gathered}
$$

et aussi

$$
\begin{gathered}
d X^{2}=\left\{X^{(x)} d x+X^{(y)} d y\right\}^{2}, \\
\left.d X d Y=\left\{X^{(x)} d x+X^{(y)} d y\right\} \mid Y^{(x)} d x+Y^{(y)} d y\right\} \\
d Y^{2}=\left\{Y^{(x)} d x+\left.Y^{(y)} d y\right|^{2}\right.
\end{gathered}
$$


En posant

$$
\left.\begin{array}{c}
\varphi(P, Y)=A^{\prime}+B^{\prime} r+C^{\prime \prime} s+D^{\prime} t+E^{\prime \prime}\left(r t-s^{2}\right) \equiv \varphi(X, Y) R, \\
\varphi(X, P)=A^{\prime \prime}+B^{\prime \prime} r+C^{\prime \prime} s+D^{\prime \prime} t+E^{\prime \prime}\left(r t-s^{2}\right) \equiv \varphi(X, Y) S, \\
\varphi(X, Q)=A^{\prime \prime \prime}+B^{\prime \prime \prime} r+C^{\prime \prime \prime} s+D^{\prime \prime \prime} t+E^{\prime \prime}\left(r t-s^{2}\right) \equiv \varphi(X, Y) T, \\
K^{\prime \prime \prime} P Q-K^{\prime \prime}\left(1+Q^{2}\right)=K_{1}, \\
K^{\prime \prime \prime}\left(1+P^{2}\right)-K^{\prime}\left(1+Q^{2}\right)=K_{2}, \\
K^{\prime \prime}\left(1+P^{2}\right)-K^{\prime} P Q=K_{3},
\end{array}\right\}
$$

et remarquant en passant que on peut avoir les équations simultanées

le déterminant

$$
K_{1}=K_{2}=K_{3}=0 \text {, }
$$

$$
\left|\begin{array}{ccc}
0 & 1+P^{2} & -P Q \\
1+P^{2} & 0 & -\left(1+Q^{2}\right) \\
P Q & -\left(1+Q^{2}\right) & 0
\end{array}\right|
$$

étant égal à zéro identiquement, on trouve pour la fonction $\Omega$ la forme suivante

$$
\begin{gathered}
\Omega=\left\{M_{1}\left(Y^{(x)}\right)^{2}+M_{2} X^{(x)} Y^{(x)}+M_{3}\left(X^{(x)}\right)^{2}\right\} d x^{2} \\
+\mid 2 M_{1} Y^{(x)} Y^{(y)}+M_{2}\left(X^{(x)} Y^{(y)}+X^{(y)} Y^{(x)}\right)+ \\
\left.+2 M_{3} X^{(x)} X^{(y)}\right\} d x d y \\
\text { où } \quad+\left\{M_{1}\left(Y^{(y)}\right)^{2}+M_{2} Y^{(y)} X^{(y)}+M_{3}\left(X^{(y)}\right)^{2}\right\} d y^{2} \\
M_{i}=A_{i}+B_{i} r+C_{i} s+D_{i} t+E_{i}\left(r t-s^{2}\right) .
\end{gathered}
$$

Done on aura

où

$$
\Omega=\left(\varphi_{1} r+\varphi_{4}\right) d x^{2}+\left(2 \varphi_{2} s+\varphi_{5}\right) d x d y+\left(\varphi_{3} t+\varphi_{8}\right) d y^{2},
$$

$$
\begin{gathered}
\varphi_{1}=2 X^{x} X_{p} A+X^{x^{2}} B+\left(X_{p}^{2} A+2 X^{x} X_{p} B_{3}\right) r+ \\
+\left(2 X_{p} X_{q} A+2 X^{x} X_{q} B+2 X^{x} X_{p} C\right) s+ \\
+\left(2 X^{\infty} X_{p} D+X^{x^{2}} E\right) t \\
+X_{p}^{2} B r^{2}+\left(2 X_{p} X_{q} \dot{B}+X_{p}^{2} C\right) r s+ \\
+\left(X_{p}^{2} D+2 X^{x} X_{p} E\right) r t+\left(2 X_{p} X_{q} D+2 X^{x} X_{q} E\right) s t \\
+\left(X_{q}^{2} B+2 X_{p} X_{q} C-2 X^{s} X_{p} E\right) s^{2}+ \\
+\left(X_{p}^{2} E r+2 X_{p} X_{q} E s\right)\left(r t-s^{2}\right)+X_{q}^{2} E s^{2} t
\end{gathered}
$$




$$
\begin{aligned}
& 2 \varphi_{2}=\left(2 X^{x} X_{p}+2 X^{y} X_{q}\right) A+2 X^{x} X^{y} C+ \\
& +\left\{2 X_{p}^{2} A+\left(2 X^{x} X_{p}+2 X^{y} X_{q}\right) B+2 X^{y} X_{p} C \nmid r\right. \\
& +\left|2 X_{p} X_{q} A+\left(2 X^{x} X_{p}+2 X^{y} X_{q}\right) C-2 X^{x} X^{y} E\right| s+ \\
& +\left\{2 X_{q}^{2} A+2 X^{x} X_{q} C+\left(2 X^{x} X_{p}+2 X^{y} X_{q}\right) D\right\} t \\
& +2 X_{p}^{2} B r^{2}+\left(2 X_{p} X_{q} B+2 X_{p}^{2} C-2 X^{y} X_{p} E\right) r s+ \\
& +\left\{X_{q}^{2} B+2 X_{p} X_{q} C+2 X_{p}^{2} D+\right. \\
& +\left(2 X^{x} X_{p}+2 X^{y} X_{q}\right) E \mid r t \\
& \left.+\mid 2 X_{p} X_{q} C-\left(2 X^{x} X_{p}+2 X^{y} X_{q}\right) E\right\} s^{2}+ \\
& +\left\{2 X_{q}^{2} C+2 X_{p} X_{q} D-2 X^{\infty} X_{q} E\right\} s t+2 X_{q}^{2} D t^{2} \\
& +\left\{2 X_{p}^{2} E r+2 X_{p} X_{q} E s+2 X_{q}^{2} E t\right\}\left(r \cdot t-s^{2}\right) \text {; } \\
& \varphi_{3}=2 X^{y} X_{q} A+X^{y^{2}} D+\left(2 X^{y} X_{q} B+X^{y^{3}} E\right) r+ \\
& +\left(2 X_{p} X_{q} A+2 X^{y} X_{q} C+2 X^{y} X_{p} D\right) s \\
& +\left(X_{q}^{2} A+2 X^{y} X_{q} D\right) t+\left(2 X_{p} X_{q} B+2 X^{y} X_{p} E\right) r s+ \\
& +\left(2 X_{q}^{2} B+2 X^{y} X_{q} E\right) \cdot t \\
& +\left(2 X_{p} X_{q} C+X_{p}^{2} D-2 X^{y} X_{q} E\right) s^{2}+\left(X_{q}^{2} C+2 X_{p} X_{q} D\right) s t+ \\
& +X_{q}^{2} E t^{2}+X_{p}^{q} E r s^{2}+\left(2 X_{p} X_{q} s+X_{q}^{q} t\right) E\left(v t-s^{2}\right) \text {; } \\
& \varphi_{4}=X^{x^{2}} A+\left(2 X^{x} X_{q} A+X^{x^{2}} C\right) s+X^{x^{2}} D t+ \\
& +\left(X_{q}^{2} A+2 X^{x} X_{q} C-X x^{2} E\right) s^{2}+2 X^{x} X_{q} D s t \\
& +\left(X_{q}^{2} C-2 X^{x} X_{q}\right) s^{3}+X_{q}^{2} D s^{2} t-X_{q}^{2} E s^{4} \text {; } \\
& \varphi_{5}=2 X^{x} X^{y} A+\left(2 X^{y} X_{p} A+2 X^{x} X y B\right) r^{*}+ \\
& +\left(2 X^{x} X_{q} A+2 X^{x} X^{y} D\right) t \\
& +\left(2 X_{p} X_{q} A+2 X^{x} X_{q} B+2 X^{y} X_{p} D+2 X^{x} X^{y} E\right) r t+ \\
& +2 X y X_{p} B r^{2}+2 X^{\infty} X_{q} D t^{2} \\
& +\left(2 X_{p} X_{q} D+2 X^{x} X_{q} E\right) r t^{2}+\left(2 X_{p} X_{q} B+2 X y X_{p} E\right) r^{2} t+ \\
& \text { +ै } 2 X_{p} X_{q} E r^{2} t^{2} \\
& \varphi_{6}=X y^{2} A+X y^{2} B r+\left(2 X y X_{p} A+X y^{2} C\right) s+2 X y X_{p} B r s+ \\
& +\left(X_{p}^{2} A+2 X y X_{p} C-X y^{2} E\right) s^{2} \\
& +X_{p}^{2} B r s^{2}+\left(X_{p}^{2} C-2 X y X_{p}\right) s^{3}-X_{p}^{2} \boldsymbol{E} s^{4} \text {; }
\end{aligned}
$$


où

$$
\begin{aligned}
& X^{x^{2}} K \equiv X^{x^{9}} K_{3}+X^{x} Y^{x} K_{2}+Y^{x^{2}} K_{1}, \\
& X_{p}^{2} K \equiv X_{p}^{2} K_{3}+X_{p} Y_{p} K_{2}+Y_{p}^{2} K_{1}, \\
& X^{y^{\mathrm{s}}} K \equiv X^{y^{\mathrm{a}}} K_{3}+X y Y^{y} K_{2}+Y^{y^{2}} K_{1} \text {, } \\
& X_{q}^{2} K \equiv X_{q}^{2} K_{3}+X_{q} Y_{q} K_{2}+Y_{q}^{2} K_{1}, \\
& 2 X^{x} X^{y} K \equiv 2 X^{x} X^{y} K_{s}+\left(X^{x} Y^{y}+X^{y} Y^{x}\right) K_{2}+2 Y^{x} Y^{y} K_{1} \text {, } \\
& 2 X_{p} X_{q} K \equiv 2 X_{p} X_{q} K_{3}+\left(X_{p} Y_{q}+X_{q} Y_{p}\right) K_{2}+2 Y_{p} Y_{q} K_{1} \text {, } \\
& 2 X^{y} X_{p} K \equiv 2 X^{y} X_{p} K_{3}+\left(X^{y} Y_{p}+X_{p} Y^{y}\right) K_{2}+2 Y^{y} Y_{p} K_{1} \text {, } \\
& 2 X^{x} X_{q} K \equiv 2 X^{x} X_{q} K_{3}+\left(X^{\infty} Y_{q}+X_{q} Y^{x}\right) K_{2}+2 Y^{\infty} Y_{q} K_{1} \text {, } \\
& 2\left(X^{x} X_{p} \pm X^{y} X_{q}\right) K \equiv 2\left(X^{x} X_{p} \pm X y X_{q}\right) K_{3}+ \\
& +\left(X^{x} Y_{p}+X_{p} Y^{x} \pm X^{y} Y_{q} \pm X_{q} Y y\right) K_{2}+ \\
& +2\left(Y^{x} Y_{p} \pm Y^{y} Y_{q}\right) K_{1} \text {. }
\end{aligned}
$$

En comparant l'équation

avec l'équation (5) on trouve

$$
\Omega=0
$$

$$
\varphi(X, Y)=1=0, \quad \varphi_{1}=\varphi_{2}=\varphi_{3}=0, \quad \varphi_{4}^{\prime}=\varphi_{5}=\varphi_{6}=0
$$

pour toutes les valeurs des quantités $r, s, t$; ce qui donne les équations suivantes pour obtenir les formes des fonctions $X, Y, P, Q$ :

$$
\begin{array}{rrrr}
X^{x^{2}} A=0,(21) & X_{p}^{2} E=0,(24) & X^{x^{2}} D=0,(27) & X y^{2} B=0,(30) \\
2 X^{x} X^{y} A=0,(22) & 2 X_{p} X_{q} E=0,(25) & 2 X^{\infty} X_{q} D=0,(28) & 2 X^{y} X_{p} B=0,(31) \\
X^{y^{2}} A=0,(23) & X_{q}^{2} E=0,(26) & X_{q}^{2} D=0,(29) & X_{p}^{2} B=0,(32) \\
2 X^{y} X_{p} A+2 X^{x} X^{y} B=0,(33) & 2 X_{p} X_{q} B+2 X^{y} X_{p} E=0,(38) \\
2 X^{x} X_{q} A+X^{x^{2}} C= & 0,(34) \\
& 2 X^{x} X_{q} C+2\left(X^{x} X_{p}-X^{y} X_{q}\right) D=0,(39) \\
2 X^{y} X_{p} A+X^{y^{2}} C= & 0,(35) \\
& X_{p}^{2} C-2 X^{y} X_{p} E=0,(40) \\
2 X^{x} X_{q} A+2 X^{x} X^{y} D=0,(36) & X_{q}^{2} C-2 X^{x} X_{q} E=0,(41) \\
2\left(X^{x} X_{p}-X^{y} X_{q}\right) B- & 2 X^{y} X_{p} C=0,(37) \\
& 2 X_{p} X_{q} D+2 X^{x} X_{q} E=0,(42)
\end{array}
$$




$$
\begin{gathered}
2\left(X^{x} X_{p}-X^{y} X_{q}\right) A+2 X^{x^{q}} B-2 X^{x} X^{y} C=0 \\
2\left(X^{x} X_{p}-X^{y} X_{q}\right) A+2 X^{x} X^{y} C-2 X^{y^{2}} D=0 \\
X_{p}^{2} A+2 X^{y} X_{p} C-X^{y^{2}} E=0 \\
X_{q}^{2} A+2 X^{x} X_{q} C-X^{x^{2}} E=0 \\
2 X_{q}^{2} B+2 X_{p} X_{q} C-2\left(X^{x} X_{p}-X^{y} X_{q}\right) E=0 \\
2 X_{p} X_{q} C+2 X_{p}^{2} D+2\left(X^{x} X_{p}-X^{y} X_{q}\right) E=0 \\
2 X_{p} X_{q} A+4 X^{x} X_{q} B+2\left(X^{x} X_{p}-X^{y} X_{q}\right) C+2 X^{x} X^{y} E=0 \\
2 X_{p} X_{q} A+2 X^{x} X_{q} B+2 X^{y} X_{p} D+2 X^{x} X^{y} E=0 .
\end{gathered}
$$

Il faut observer que les équations (43) et (44) ne peurent pas être égales à zéro identiquement parce que elles résultent des termes du degré zéro des différences

$$
\varphi_{1}-\varphi_{2}, \quad \varphi_{2}-\varphi_{3} .
$$

La détermination des formes des fonctions $X, Y, P, Q$ au moyen de ces équations de condition est assez difficile, cependant on peut résoudre le problème indirectement. Nous avons déterminé dans une Note précédente les transformations de contact entre les lignes droites et les sphères, et LiE a remarqué que toutes les transformations qui changent les lignes asymptotiques en lignes de courbure transforment aussi les lignes droites en sphères, done pour trouver les transformations entre les lignes asymptotiques et les lignes de courbure il fant employer seulement les operations de différentiation et de substitution. Le procédé est simple mais tres-laborieux, et il faut réserver les résultats de cette computation longue pour une autre communication. On conclut néanmoins facilement au résultat que les fonctions $X, Y, P, Q$ définies par les équations

$$
\begin{gathered}
\omega_{1}=\sum_{1}^{4} \dot{\Sigma}_{j}\left(a_{1 j} X+i a_{1 j} Y+a_{2 j} Z+a_{3 j}\right) x_{j}, \\
\omega_{2}=\sum_{1}^{4}{ }_{j}\left(a_{2 j} X-i a_{9 j} Y-a_{1 j} Z+a_{4 j}\right) x_{j}, \\
x_{1}=x, \quad x_{2}=y, \quad x_{3}=z, \quad x_{4}=1, \quad i=\sqrt{-1}, \\
\frac{\omega_{1}^{x}}{\omega_{3}^{x}}=\frac{\omega_{1}^{y}}{\omega_{2}^{y}}=\frac{\omega_{1}^{X}}{\omega_{2}^{X}}=\frac{\omega_{1}^{Y}}{\omega_{2}^{Y}},
\end{gathered}
$$


ou par les équations

$$
\begin{gathered}
\omega_{3}=\sum_{1}^{4}\left(a_{1 j} X-i a_{1 j} Y+a_{2 j} Z+a_{3 j}\right) x_{j}, \\
\omega_{1}=\sum_{1}^{4}\left(a_{2 j} X+i a_{2 j} Y-a_{1 j} Z+a_{4 j}\right) x_{j}, \\
x_{1}=x, \quad x_{2}=y, \quad x_{3}=z, \quad x_{4}=1, \quad i=\sqrt{-1}, \\
\frac{\omega_{3}^{x}}{\omega_{4}^{x}}=\frac{\omega_{3}^{y}}{\omega_{4}^{y}}=\frac{\omega_{3}^{X}}{\omega_{1}^{Y}}=\frac{\omega_{3}^{Y}}{\omega_{4}^{X}},
\end{gathered}
$$

constituent deux familles de $\infty^{15}$ transformations de contact qui changent les lignes asymptotiques en lignes de courbure. Chaque famille contient la transformation de LIE; la première famille la donne pour

$$
a_{14}=a_{31}=a_{33}=a_{43}=1 \text {, }
$$

et toutes les autres constantes égales à zéro; on la trouve dans la deuxième famille en posant

$$
a_{11}=-a_{24}=a_{33}=-a_{33}=1,
$$

et toutes les autres constantes égales à zéro.

Dans certains cas on peut réduire les équations de condition et en nombre et en forme. Considérons les déterminants

$$
\begin{gathered}
G \equiv\left|\begin{array}{ccc}
X^{x^{2}} & X^{x} Y^{x} & Y^{x^{3}} \\
2 X^{x} X^{y} & X^{x} Y^{y}+X^{y} Y^{x} & 2 Y^{x} Y^{y} \\
X^{y^{2}} & X^{x} Y^{y} & Y^{y^{2}}
\end{array}\right|, \\
I \equiv\left|\begin{array}{ccc}
X_{p}^{2} & X_{p} Y_{p} & Y_{p}^{2} \\
2 X_{p} X_{q} & Y_{p} Y_{q}+X_{q} Y_{p} & 2 Y_{p} Y_{q} \\
X_{q}^{2} & X_{q} Y_{q} & Y_{q}^{2} \\
X^{x^{2}} & X^{x} Y^{x} & Y^{x^{2}} \\
2 X^{x} Y_{q} & X^{x} Y_{q}+X_{q} Y^{x} & 2 Y^{x} Y_{q} \\
X_{y}^{2} & X_{q} Y_{q} & Y_{q}^{2} \\
X^{2} & X^{2} Y^{y} & Y^{3} \\
2 X^{y} X_{p} & X y Y_{p}+X_{p} Y y & 2 Y^{y} Y_{p} \\
X_{p}^{2} & X_{p} Y_{p} & Y_{p}^{2}
\end{array}\right|,
\end{gathered}
$$


Les cas suivants s'excluent

$$
\begin{aligned}
& \text { 1. } G=1=0, I I=0, I=0, J=0 ; \\
& \text { 2. } .^{\circ} G=0, H=0, I=0, J=0 \text {; } \\
& \text { 3. }{ }^{\circ} G=1=0, H=0, I=\mid=0, J=1=0 \text {; } \\
& \text { 4. } \quad G=0, H=1=6, I=0, J=0 \text {; } \\
& \text { 5. } G=0, H=0, I=0, J=0 \text {; } \\
& \text { 6. } G=0, H=1=0, I=0, J=1=0 \text {; } \\
& \text { 7. } G=0, H=0, I=i=0, J=0 \text {; } \\
& \text { 8. } \quad G=1=0, H=0, I=0, J=0 \text {; } \\
& \text { 9. } \quad G=0, H=0, I==0, J=0 \text {; }
\end{aligned}
$$

en effet si on $J==0$, il existaient les trois équations

$$
B_{1}=B_{2}=B_{3}=0 \text {; }
$$

de plus les équations (33), (35), (37), (38) et (40) donnent

done on a

$$
X y^{2} C=2 X y X_{p} C=X_{p}^{2} C=0 \text {; }
$$

$$
C_{1}=C_{2}=C_{3}=0 \text {; }
$$

de ces équations-ci et des équations $(43),(44),(49),(50),(47)$ et (48) on trouve

donc

$$
X y^{2} D=2 X y X_{p} D=X_{p}^{2} D=0 \text {; }
$$

Mais les équations

$$
D_{1}=D_{2}=D_{3}=0 \text {. }
$$

$$
B_{1}=B_{a}=B_{3}=C_{1}=C_{2}=C_{3}=D_{1}=D_{2}=D_{3}=0
$$

et les équations

$$
A_{1}=A_{2}=A_{3}=E_{1}=E_{2}=E_{3}=0
$$

ne peuvent pas exister simultanément; car si toutes les formes $A_{1}, A_{2}, A_{3}$ etaient égales à zéro, les équations (43) et (44) donneraient

$$
\varphi_{1}-\varphi_{2}=\varphi_{2}-\varphi_{3}=0 \text {. }
$$

D'ailleurs si nous avons

$$
J=1=0, \quad E_{1}=E_{2}=E_{3}=B_{1}=B_{2}=B_{3}=C_{1}=C_{2}=C_{3}=D_{1}=D_{2}=D_{3}=0,
$$


les équations (23), (33) et (45) donnent

done

$$
X y^{3} A=2 X y X_{p} A=X_{p}^{2} A=0 ;
$$

$$
A_{1}=A_{\mathrm{g}}=A_{3}=0 ;
$$

ainsi toutes les quantités $E_{1}, E_{2}, E_{3}$ ne sont égales à zéro et on a

$$
G=H=0 \text {; }
$$

donc les cas $1 .^{\circ}, 2 .^{\circ}, 3 .^{\circ}, 4 .^{\circ}, 5 .^{\circ}, 6 .^{\circ}$ disparaissent.

Maintenant supposons que $I==0$; ainsi

$$
D_{1}=D_{2}=D_{3}=0 \text {; }
$$

des équations (34), (36), (39), (41) et (42) on trouve

done

$$
X^{x^{2}} C=2 X^{x} X_{q} C=X_{q}^{2} C=0 \text {; }
$$

$$
C_{1}=C_{2}=C_{8}=0 \text {; }
$$

encore des équations (43), (44), (49), (50), (47) et (48) on a

qui demandent que

$$
X^{x^{2}} B=2 X^{x} X_{q} B=X_{q}^{2} B=0 \text {, }
$$

$$
B_{1}=B_{2}=B_{3}=0 \text {. }
$$

Outre cela on observe que, si on a

$$
I==0, \quad E_{1}=E_{\mathrm{a}}=E_{3}=0,
$$

on trouvait des équations $(21),(34)$, et $(46)$

$$
A_{1}=A_{2}=A_{3}=0
$$

ce qui n'est pas possible; ainsi si on a $I=0$, on a aussi les équations

$$
G=H=0 \text {, }
$$

et les cas $7 .^{\circ}, 8 .^{\circ}, 9 .^{\circ}$ s'excluent. Ils restent les sept cas

$$
\begin{array}{ll}
10 .^{\circ} & G=0, H=0, I=0, J=0 ; \\
11 .^{\circ} & G=1=0, H=0, I=0, J=0 ; \\
12 .^{\circ} & G=0, H=1=0, I=0, J=0 ; \\
13 .^{\circ} & G=0, H=0, I=1=0, J=0 ; \\
14 .^{\circ} & G=0, H=0, I=0, J=\mid=0 ; \\
1 .^{\circ} & G=0, H=0, I=\mid=0, J=1=0 ; \\
16 .^{\circ} & G=1=0, H=\mid=0, I=0, J=0 .
\end{array}
$$


Considérons encore les deux cas particuliers suivants

$$
\begin{aligned}
& B_{1}=B_{2}=B_{3}=C_{1}=C_{2}=C_{3}=D_{1}=D_{2}=D_{3}=0 ; \\
& A_{1}=A_{2}=A_{3}=C_{1}=C_{2}=C_{3}=E_{1}=E_{2}=E_{3}=0 ;
\end{aligned}
$$

pour le premier cas on a aussi les équations

$$
\begin{gathered}
X^{x^{\prime}} A=2 X^{x} X^{y} A=X^{y^{y}} A=2 X^{x} X_{q} A=2 X^{y} X_{p} A= \\
=2\left(X^{x} X_{p}-X^{y} X_{q}\right) A=0 \\
\begin{array}{r}
X_{y}^{2} E=2 X_{p} X_{q} E=X_{q}^{2} E=2 X^{x} X_{q} E=2 X^{y} X_{p} E= \\
=2\left(X^{x} X_{p}-X^{y} X_{q}\right) E=0 \\
X_{p}^{2} A-X^{y^{2}} E=2 X_{p} X_{q} A+2 X^{x} X^{y} E=X_{q}^{2} A-X^{x^{2}} E=0
\end{array}
\end{gathered}
$$

pour le deuxième cas on a des équations tout-à-fait semblables.

Le premier cas donne une démonstration analytique de la propriété de la transformation de LiE. En effet, les équations définissantes de la transformation de Lie sont

donc on a

$$
\begin{gathered}
X+i Y=-z-x \frac{p x+q y}{q-x}, \quad X-i Y=\frac{p+y}{q-x}, \quad Z=\frac{p x+p y}{q-x}, \\
P=\frac{q x-1}{q+x}, \quad Q=-i \frac{q x+1}{q+x}
\end{gathered}
$$

$$
\begin{aligned}
& X^{x}=\frac{1}{2} \frac{(p+y)\left(1-q^{2}\right)}{(q-x)^{2}}, \quad X y=\frac{1}{2} \frac{1-q^{2}}{q-x}, \\
& X_{p}=\frac{1}{2} \frac{1-x^{2}}{q-x}, \quad X_{q}=-\frac{1}{2} \frac{\left(1-x^{2}\right)(p+y)}{(q-x)^{2}}, \\
& Y^{x}=\frac{i}{2} \frac{(p+y)\left(1+q^{2}\right)}{(q-x)^{2}}, \quad Y y=\frac{i}{2} \frac{1+q^{2}}{q-x}, \\
& Y_{p}=\frac{i}{2} \frac{\left(1+x^{2}\right)}{q-x}, \quad Y_{q}=-\frac{i}{2} \frac{\left(1+x^{2}\right)(p+y)}{(q-x)^{2}}, \\
& P^{x}=\frac{1+q^{2}}{(q+x)^{2}}, \quad P^{y}=0, \quad P_{p}=0, \quad P_{q}=\frac{1+x^{2}}{(q+x)^{2}}, \\
& Q^{x}=\frac{i\left(1-q^{2}\right)}{(q+x)^{2}}, \quad Q^{y}=0, \quad Q_{p}=0, \quad Q_{q}=\frac{\left(1-x^{2}\right)\left(q^{2}-1\right)}{(q+x)^{2}} .
\end{aligned}
$$

En posant

$$
\begin{aligned}
& f A^{\prime}=\alpha^{\prime}, f A^{\prime \prime}=\alpha^{\prime \prime}, f A^{\prime \prime \prime}=\alpha^{\prime \prime \prime}, f B^{\prime}=\beta^{\prime}, f B^{\prime \prime}=\beta^{\prime \prime}, f B^{\prime \prime \prime}=\beta^{\prime \prime \prime}, \\
& \\
& f C^{\prime}=\gamma^{\prime}, f C^{\prime \prime}=\gamma^{\prime \prime}, f C^{\prime \prime \prime}=\gamma^{\prime \prime \prime}, \\
& g D^{\prime}=\delta^{\prime}, \quad g D^{\prime \prime}=\delta^{\prime \prime}, \quad g D^{\prime \prime \prime}=\delta^{\prime \prime \prime}, f E^{\prime}=\varepsilon^{\prime}, f E^{\prime \prime}=\varepsilon^{\prime \prime}, \quad f E^{\prime \prime \prime}=\varepsilon^{\prime \prime \prime},
\end{aligned}
$$


où

nous avons

$$
f=2(q-x)(q+x)^{2}, \quad g=\frac{2(q-x)^{2}(q+x)^{2}}{p+y},
$$

$$
\begin{array}{cl}
\alpha^{\prime}=i\left(1+q^{2}\right)^{2}, & \alpha^{\prime \prime}=q^{4}-1, \quad \alpha^{\prime \prime \prime}=-i\left(q^{2}-1\right)^{2}, \\
& \beta^{\prime}=\beta^{\prime \prime}=\beta^{\prime \prime \prime}=0, \\
\gamma^{\prime}=i\left(1+x^{2}\right)\left(1+q^{2}\right), & \gamma^{\prime \prime}=q^{2} x^{2}-1, \quad \gamma^{\prime \prime \prime}=-i\left(1-x^{2}\right)\left(1-q^{2}\right), \\
\delta^{\prime}=-i\left(1+x^{2}\right)\left(1+q^{2}\right), & \delta^{\prime \prime}=1-q^{2} x^{2}, \quad \delta^{\prime \prime \prime}=i\left(1-x^{2}\right)\left(1-q^{2}\right), \\
\varepsilon^{\prime}=-i\left(1+x^{2}\right)^{2}, & \varepsilon^{\prime \prime}=1-x^{4}, \quad \varepsilon^{\prime \prime \prime}=i\left(1+x^{2}\right)^{2}
\end{array}
$$

done

$$
\begin{array}{cc}
A_{1}=-\frac{1}{2} \frac{\left(1-q^{2}\right)^{2}}{(q+x)^{3}}, \quad A_{2}=\frac{i\left(1-q^{4}\right)}{(q+x)^{3}}, & A_{3}=\frac{1}{2} \frac{\left(1+q^{2}\right)^{2}}{(q+x)^{3}}, \\
E_{1}=-\frac{1}{2} \frac{\left(1-x^{2}\right)^{2}}{(q+x)^{3}}, \quad E_{2}=\frac{i\left(1-x^{4}\right)}{(q+x)^{3}}, & E_{3}=\frac{1}{2} \frac{\left(1+x^{2}\right)^{2}}{(q+x)^{3}}, \\
B_{1}=B_{2}=B_{3}=C_{1}=C_{2}=C_{3}=D_{1}=D_{2}=D_{3}=0,
\end{array}
$$

et nous voyons sans difficulté que les autres équations de condition sont satisfaites et ensuite que la transformation de LiE changent les lignes asymptotiques en lignes de courbure.

On remarque en passant qu'on peut attendre la desparition de plusieurs déterminants des équations du système particulier (iii); trois de ces déterminants se réduisent aux formes simples, savoir

$$
\begin{aligned}
& \left(\lambda_{1}, \mu_{1}, \nu_{1}\right)=4\left|\begin{array}{cc}
X^{x} & X^{y} \\
Y^{x} & Y^{y}
\end{array}\right| \cdot\left|\begin{array}{cc}
X^{x} & X_{p} \\
Y^{x} & Y_{p}
\end{array}\right| \cdot\left|\begin{array}{cc}
X^{y} & X_{q} \\
Y^{y} & Y_{q}
\end{array}\right|, \\
& \left(\lambda_{2}, \mu_{y}, \nu_{2}\right)=4\left|\begin{array}{cc}
X_{p} & Y_{p} \\
X_{q} & Y_{q}
\end{array}\right| \cdot\left|\begin{array}{cc}
X^{\infty} & X_{p} \\
Y^{x} & Y_{p}
\end{array}\right| \cdot\left|\begin{array}{cc}
X^{y} & X_{q} \\
Y^{y} & Y_{q}
\end{array}\right| \text {, } \\
& \left(\lambda_{3}, \mu_{3}, \nu_{3}\right)=4[X, Y] \quad\left|\begin{array}{cc}
X^{x} & X^{y} \\
Y^{x} & Y^{y}
\end{array}\right| \cdot\left|\begin{array}{cc}
X_{p} & Y_{p} \\
X_{q} & Y_{q}
\end{array}\right| \text {, }
\end{aligned}
$$

où

$$
\begin{array}{r}
\lambda_{1}=2 X^{\infty} X^{y}, \quad \mu_{1}=X^{x} Y^{y}+X^{y} Y^{x}, \\
\nu_{1}=2 Y^{x} Y^{y}, \\
\lambda_{2}=2 X_{p} X_{q}, \quad \mu_{2}=X_{p} Y_{q}+X_{q} Y_{p}, \\
\nu_{2}=2 Y_{p} Y_{q},
\end{array}
$$




$$
\begin{gathered}
\lambda_{3}=2\left(X^{x} X_{p}-X^{y} X_{q}\right), \quad \mu_{3}=X^{x} Y_{p}+X_{p} Y^{x}-X^{y} Y_{q}-X_{q} Y^{y}, \\
\nu_{3}=2\left(Y^{x} Y_{p}-Y^{y} Y_{q}\right), \\
\left(\lambda_{i}, \mu_{i}, \nu_{i}\right)=\left|\begin{array}{ccc}
\lambda_{i} & \mu_{i} & y_{i} \\
2 X^{y} X_{p} & X_{p} Y^{y}+X^{y} Y_{p} & 2 Y^{y} Y_{p} \\
2 X^{x} X_{q} & X_{q} Y^{x}+X^{x} Y_{q} & 2 Y^{x} Y_{q}
\end{array}\right| ;
\end{gathered}
$$

pour la transformation de LiE on a

$$
\left(\lambda_{1}, \mu_{1}, \nu_{1}\right)=\left(\lambda_{2}, \mu_{2}, \nu_{2}\right)=\left(\lambda_{3}, \mu_{3}, \nu_{3}\right)=0,
$$

parce que on a les équations

$$
\left|\begin{array}{cc}
X^{x} & X^{y} \\
Y^{x} & Y^{y}
\end{array}\right|=\left|\begin{array}{cc}
X_{p} & Y_{p} \\
X_{q} & Y_{q}
\end{array}\right|=0 .
$$

Pour une transformation de contact quelconque on a

$$
[X Y]=0 ;
$$

donc la forme du déterminant $\left(\lambda_{3}, \mu_{3}, \nu_{3}\right)$ montre qu'on a

$$
\left|\begin{array}{ccc}
X^{y} X_{p} & X^{y} Y_{p}+X_{p} Y^{y} & Y^{y} Y_{p} \\
X^{x} X_{p}-X^{y} X_{q} & X^{x} Y_{p}+X_{p} Y^{x}-X^{y} Y_{q}-X_{q} Y^{y} & Y^{x} Y_{p}-Y^{y} Y_{q} \\
X^{x} X_{q} & X^{x} Y_{q}+X_{q} Y^{x} & Y^{x} Y_{q}
\end{array}\right|=0
$$

pour une transformation de contact quelconque.

Encore on observe que les quantités $A_{1}, A_{2}, A_{3}, E_{1}, E_{2}, E_{3}$ dans la transformation de Lie satisfaient à la relation

$$
K_{2}^{3}-4 K_{3} K_{1}=0 \text {; }
$$

pourque cette relation soit vraie pour les équations

$$
2 X^{y} X_{p} K_{3}=2 X^{y} X_{q} K=0
$$

il est nécessaire ct suffisant que la relation

$$
\left|\begin{array}{cc}
X^{x} & X^{y} \\
Y^{x} & Y^{y}
\end{array}\right| \cdot\left|\begin{array}{cc}
X_{p} & X_{q} \\
Y_{p} & Y_{q}
\end{array}\right| \cdot\left|\begin{array}{cc}
X^{x} & X_{p} \\
Y^{x} & Y_{p}
\end{array}\right| \cdot \mid \begin{array}{cc}
X^{y} & X_{q} \\
Y^{y} & Y_{q}
\end{array}=0
$$

ait lieu; en vertu de l'équation $\left[\begin{array}{l}X Y] \\ Y\end{array}\right]=0$, la dernière relation s'ecrit dans l'une forme et l'autre

$$
\left|\begin{array}{cc}
X^{x} & X_{p} \\
Y^{x} & Y_{p}
\end{array}\right|^{2} \cdot\left|\begin{array}{cc}
X^{x} & X^{y} \\
Y^{x} & Y^{y}
\end{array}\right| \cdot\left|\begin{array}{cc}
X_{p} & X_{q} \\
Y_{p} & Y_{q}
\end{array}\right|=\left|\begin{array}{cc}
X^{y} & X_{q} \\
Y^{y} & Y_{q}
\end{array}\right|^{2} \cdot\left|\begin{array}{cc}
X^{x} & X^{y} \\
Y^{x} & Y^{y}
\end{array}\right| \cdot\left|\begin{array}{cc}
X_{p} & X_{q} \\
Y_{p} & Y_{q}
\end{array}\right|=0 .
$$


Transformations de contact qUi Changent lies lignes asymptotiques EN LIGNES ASYMPTOTIQUES.

La forme

devient

$$
\Phi \equiv R d X^{2}+2 S d X d Y+T d Y^{2}=0
$$

où

$$
\left(\psi_{1} r+\psi_{1}\right) d x^{2}+\left(2 \psi_{2}+\psi_{0}\right) d x d y+\left(\psi_{3} t+\psi_{0}\right) d y^{2}=0
$$

$$
\psi_{1}, \psi_{2}, \psi_{3}, \psi_{1}, \psi_{5}, \psi_{6}
$$

se dérivent immédiatement des fonctions de la note précédente

$$
\varphi_{1}, \varphi_{2}, \varsigma_{3}, \varphi_{+}, \varphi_{5}, \varphi_{i},
$$

respectivement, en posant

$$
A^{\prime}, B^{\prime}, C^{\prime}, D^{\prime}, E^{\prime}, A^{\prime \prime}, B^{\prime \prime}, C^{\prime \prime}, D^{\prime \prime}, E^{\prime \prime}, A^{\prime \prime \prime}, B^{\prime \prime \prime}, C^{\prime \prime}, D^{\prime}, E^{\prime \prime \prime}
$$

au lieu de

$$
A_{3}, B_{3}, C_{3}, D_{3}, E_{3}, A_{2}, B_{3}, C_{2}, D_{2}, E_{2}, A_{1}, B_{1}, C_{1}, D_{1}, E_{1},
$$

respectivement, et en écrivant

$$
\begin{aligned}
& \mathrm{X}^{x^{2}} A \equiv X^{x^{2}} A^{\prime}+2 \mathrm{Y}^{\infty} Y^{x} A^{\prime \prime}+Y^{x^{2}} A^{\prime \prime \prime} \text {, } \\
& X_{p}^{2} A \equiv X_{p}^{2} A^{\prime}+2 X_{p} Y_{p} A^{\prime \prime}+Y_{p}^{2} A^{\prime \prime \prime} \text {, } \\
& X^{x} X^{y} A=X^{x} X^{y} A^{\prime}+\left(X^{x} Y^{y}+X^{y} Y^{x}\right) A^{\prime \prime}+Y^{x} Y^{y} A^{\prime \prime} \text {, } \\
& X_{p} X_{q} A \equiv Y_{p} X_{q} A^{\prime}+\left(X_{p} Y_{q}+X_{q} Y_{p}\right) A^{\prime \prime}+Y_{p} Y_{q} A^{\prime \prime \prime} \text {, } \\
& X y^{\prime \prime} A \equiv X y^{y} A^{\prime}+2 X^{y} Y^{y} A^{\prime \prime}+Y^{y^{2}} A^{\prime \prime \prime} \text {, } \\
& X_{q}^{2} A \equiv X_{q}^{3} A^{\prime}+2 X_{q} Y_{q} A^{\prime \prime}+Y_{q}^{2} A^{\prime \prime \prime}, \\
& X^{x} \mathrm{X}_{p} A \equiv X^{x} X_{p} A^{\prime}+\left(X^{x} Y_{p}+X_{p} Y^{x}\right) A^{\prime \prime}+Y^{\infty} Y_{p} A^{\prime \prime \prime}, \\
& X^{y} X_{p} A \equiv X^{y} X_{p} A^{\prime}+\left(X y Y_{p}+X_{p} Y^{y}\right) A^{\prime \prime}+Y^{y} Y_{p} A^{\prime \prime \prime}, \\
& \mathrm{X}^{\infty} X_{q} A \equiv X^{\infty} X_{q} A^{\prime}+\left(X^{\infty} Y_{q}+X_{q} Y^{\infty}\right) A^{\prime \prime}+Y^{\infty} Y_{q} A^{\prime \prime \prime} \text {, } \\
& X^{y} X_{q} A \equiv X^{y} X_{q} A^{\prime}+\left(X^{y} Y_{q}+X_{q} Y^{y}\right) A^{\prime \prime}+Y^{y} Y_{q} A^{\prime \prime} \text {; }
\end{aligned}
$$

Pour que l'équation $\phi=0$ soit une conséquence de l'équation

$$
r d x^{2}+2 s d x d y+t d y^{2}=0
$$


il faut que les relations

$$
\varphi(X, Y)=1=0, \quad \psi_{1}==\psi_{2}=\psi_{3}=1=0, \quad \psi_{1}=\psi_{0}=\psi_{8}=0
$$

aient lieu pour toutes les valeurs de $r, s, t$; done on trouve les équations suivantes pour les fonctions $X, Y, P, Q$ :

$$
\begin{aligned}
& X^{x^{2}} A=X^{x} X y A=X y^{2} A=0, \\
& X y^{4} B=X^{y} X_{p} B=X_{p}^{2} B=0, \\
& X^{w^{3}} D=X^{x} X_{q} D=X_{q}^{2} D=0 \text {, } \\
& X_{p}^{2} E=X_{p} X_{q} E=X_{q}^{2} E=0, \\
& X^{y} X_{p} A+X^{\infty} X^{y} B=0, \\
& X_{p} X_{q} B+X^{y} X_{p} E=0 \\
& 2 X^{\circ} X_{q} A+X^{x^{\circ}} C=0 \text {, } \\
& X^{x} X_{q} C+\left(X^{x} X_{p}-X^{y} X_{q}\right) D=0, \\
& 2 X^{y} X_{p} A+X y^{*} C=0 \text {, } \\
& X_{p}^{2} C-2 X^{y} X_{p} E=0, \\
& \left(X^{\infty} X_{p}-X^{y} X_{q}\right) B-X^{y} X_{p} C=0, \\
& X_{q}^{2} C-2 X^{x} X_{q} E=0 \text {, } \\
& X^{x} X_{g} A+X^{x} X^{y} D=0 \text {, } \\
& X_{p} X_{q} D+X^{\infty} X_{q} E=0, \\
& \left(X^{x} X_{p}-X^{y} X_{q}\right) A+X^{x^{y}} B-X^{x} X^{y} C=0 \text {, } \\
& X_{p}^{2} A+2 X^{y} X_{p} C-X^{y^{2}} E=0, \\
& \left(X^{x} X_{p}-X^{y} X_{q}\right) A+X^{x} X^{y} C-X^{y^{q}} D=0 \text {, } \\
& X_{q}^{2} A+2 X^{x} X_{q} C-X^{x^{2}} E=0, \\
& X_{q}^{2} B+X_{p} X_{q} C-\left(X^{x} X_{p}-X^{y} X_{q}\right) E=0, \\
& X_{p} X_{q} A+2 X^{x} X_{q} B+\left(X^{x} X_{p}-X^{y} X_{q}\right) C+X^{x} X^{y} E=0 \text {, } \\
& X_{p} X_{u} C+X_{p}^{2} D+\left(X^{x} X_{p}-X^{y} X_{q}\right) E=0 \text {, } \\
& X_{p} X_{q} A+X^{x} X_{q} B+X^{y} X_{p} D+X^{x} X^{y} E=0 \text {. }
\end{aligned}
$$

Soient les transformations des transformations ponctuelles; il faut d'abord que

$$
X_{p}=X_{q}=Y_{p}=Y_{q}=Z_{p}=Z_{q}=0 .
$$

Annali di Malematica, Serie III, tomo VII 
Les équations de condition se réduisent aux équations suivantes

$$
\begin{gathered}
X^{x^{2}} A=X^{x} X^{y} A=X y^{y} A=0, \quad X^{x} X^{y} B=X y^{2} B=0, \\
X^{x^{2}} C=X y^{2} C=0, \quad X^{x^{2}} D=X^{x} X y D=0, \\
X^{x^{2}} E=X^{x} X^{y} E=X^{y^{2}} E=0, \quad X^{x^{2}} B-X^{x} X y C=0, \\
X^{x} X y C-X^{y^{2}} D=0 ;
\end{gathered}
$$

d'ailleurs

$$
\begin{aligned}
& A^{\prime}=Y_{y} P_{x}-Y^{x} P_{y}, \quad B^{\prime}=Y_{y} P_{p}, \quad C^{\prime}=Y_{y} P_{q}-Y_{x} P_{p}, \\
& D^{\prime}=-Y^{x} P_{q}, \quad E^{\prime}=0 \text {, } \\
& A^{\prime \prime}=X^{x} P_{y}-X y P_{x}, \quad B^{\prime \prime}=-X y P_{p}, \quad C^{\prime \prime}=X^{x} P_{p}-X^{y} P_{q} \text {, } \\
& D^{\prime \prime}=X^{n} P_{q}, \quad E^{\prime \prime}=0, \\
& A^{\prime \prime \prime}=X^{x} Q_{y}-X y Q^{x}, \quad B^{\prime \prime \prime}=-X^{y} Q_{p}, \quad C^{\prime \prime \prime}=X^{x} Q_{p}-X y Q_{q}, \\
& D^{\prime \prime \prime}=X^{x} Q_{q}, \quad E^{\prime \prime \prime}=0 \text {. }
\end{aligned}
$$

Il faut distinguer deux cas snivant que le déterminant

$$
\left|\begin{array}{ccc}
X^{x^{2}} & 2 X^{x} Y^{x} & Y^{x^{2}} \\
X^{x} X^{y} & X^{x} X^{y}+X^{y} Y^{x} & Y^{x} Y^{y} \\
X^{y^{3}} & 2 X^{y} Y^{y} & Y y^{q}
\end{array}\right|
$$

soit égal à zéro ou différent de zéro. Ce déterminant est égal à

$$
\Delta^{3} \equiv\left|\begin{array}{cc}
X^{x} & X^{y} \\
Y^{x} & Y^{y}
\end{array}\right|^{3} \equiv-\left|\begin{array}{ccc}
X_{x} & X_{y} & X_{z} \\
Y_{x} & Y_{y} & Y_{z} \\
p & q & -1
\end{array}\right|^{3}
$$

Considérons le cas où $\Lambda=0$; en observant que pour une transformation ponctuelle quelconque

où

$$
\pi(X, Y) P==\pi(Z, Y), \quad \pi(X, Y) Q=\pi(X, Z)
$$

$$
\pi(M, N) \equiv\left|\begin{array}{ccc}
M_{\infty} & M_{y} & M_{z} \\
N_{x} & N_{y} & N_{z} \\
p & q & -1
\end{array}\right|,
$$

on voit que les fonctions $P$ et $Q$ deviennent ou infinies ou indéterminées; 
done on peut avoir seulement

il suit que

$$
\Delta=0
$$

$$
Y^{y} P^{x}-Y^{x} P^{y}=X^{x} P^{y}-X^{y} P^{x}=X^{x} Q^{y}-X^{y} Q^{x}=0 ;
$$

c'est-à-dire

d'ailleurs des équations

$$
\frac{X^{x}}{X^{y}}=\frac{Y^{x}}{Y^{y}}=\frac{P^{x}}{P^{y}}=\frac{Q^{x}}{Q^{y}}
$$

$$
[P, Y]=[Q, X]=0
$$

on trouve, dans le cas de transformations ponctuelles

mais les relations

$$
\frac{X^{x}}{\bar{X}^{y}}=-\frac{Q_{q}}{Q_{p}}, \quad \frac{Y^{x}}{Y^{y}}=-\frac{P_{q}}{P_{p}} ;
$$

$$
\frac{X^{x}}{X^{y}}=-\frac{P_{q}}{P_{p}}, \quad \frac{Y^{x}}{Y^{y}}=-\frac{Q_{q}}{Q_{p}}
$$

contredisent les relations

$$
[P, X]=[Q, Y]=\rho=\mid=0 ;
$$

donc, si $\Delta==0$, on a ou

$$
X^{x}=X^{y}=Y^{x}=Y^{y}=0
$$

ou

$$
P^{x}=P^{y}=Q^{x}=Q_{y}=0 ；
$$

mais l'evanouissement de $X^{x}, X^{y}, Y^{x}$ et $Y^{y}$ est incompatible avec le nonévanouissement du déterminant $\Delta$; donc on conclut au résultat, si

donc

$$
X_{p}=X_{q}=Y_{p}=Y_{q}=Z_{p}=Z_{q}=0
$$

$$
P^{x}=P^{y}=Q^{x}=Q^{y}=0 ;
$$

c'est-à-dire, d'après les résultats d'une note précédente, les transformations changent les plans en plans; donc les seules transformations ponctuelles qui changent les lignes asymptotiques en lignes asymptotiques sont les transformations du groupe projectif. 
Réciproquement, d'une étude semblable du déterminant

on trouve que

$$
\left|\begin{array}{ccc}
X_{p}^{2} & 2 X_{p} X_{q} & X_{q}^{2} \\
X_{p} X_{q} & X_{p} Y_{q}+X_{q} Y_{p} & Y_{p} Y_{q} \\
Y_{p}^{2} & 2 Y_{p} Y_{q} & Y_{q}^{2}
\end{array}\right|
$$

$$
X_{p}=Y_{p}=X_{q}=X_{q}=0
$$

est une conséquence de

$$
P^{x}=P^{y}=Q^{x}=Q^{y}=0,
$$

et des équations de condition.

Considérons maintenant les trois déterminants

$$
L=\left|X^{x}, Y^{y}\right|, \quad M=\left|X_{p}, Y_{q}\right|, \quad N=\left|P_{p}, Q_{q}\right| ;
$$

on verifie facilement que les cas

$$
\begin{array}{ll}
L=0, M=0, N=0 ; & L=\prime=0, M=1=0, N=0 ; \\
L=1=0, M=1=0, N=1=0 ; & L=0, M=0, N=1=0 ;
\end{array}
$$

donnent des contredictions avec les équations définissantes d'une transformation de contact.

Les cas

donnent

$$
L=1=0, M=0, N=|=0 ; \quad L=|=0, M=0, N=0 ;
$$

$$
X_{p}=X_{q}=Y_{p}=Y_{q}=P^{x}=P^{y}=Q^{x}=Q^{y}=0,
$$

et de ces relations on a

$$
Z_{p}=Z_{q}=0 .
$$

Des cas

$$
L=0, M=0, N=|=0 ; \quad L=0, M=|=0, N=0 ;
$$

on trouve ou

ou

$$
X_{p}=X_{q}=Y_{p}=Y_{q}=0,
$$

$$
P_{p}=P_{q}=Q_{p}=Q_{q}=0 ;
$$

le premier cas donne des transformations projectives. Pour le deuxième 
cas on a

$$
\begin{array}{ll}
{[Y, P]=Y_{p} P^{x}+Y_{q} P^{y}=0,} & {[X, Q]=X_{p} Q^{x}+X_{q} Q^{y}=0,} \\
{[X, P]=X_{p} P^{x}+X_{q} P^{y}=\rho=1=0,} & {[Y, Q]=Y_{p} Q^{x}+Y_{q} Q^{y}=\rho=1=0 ;}
\end{array}
$$

encore

$$
\begin{array}{ll}
A^{\prime}=Y^{y} P^{x}-Y^{x} P^{y}, \quad B^{\prime}=-Y_{p} P^{y}, \quad C^{\prime}=Y_{p} P^{x}-Y_{q} P^{y}, \\
D^{\prime}=Y_{q} P^{x}, \quad E^{\prime}=0, \\
A^{\prime \prime}=X^{x} P^{y}-X^{y} P^{x}, \quad B^{\prime \prime}=X_{p} P^{y}, \quad C^{\prime \prime}=X_{q} P^{y}-X_{p} P^{x}, \\
D^{\prime \prime}=-X_{q} P^{x}, \quad E^{\prime \prime}=0, \\
A^{\prime \prime \prime}=X^{x} Q^{y}-X^{y} Q^{x}, \quad B^{\prime \prime}=X_{p} Q^{y}, \quad C^{\prime \prime}=X_{q} Q^{y}-X_{p} Q^{x} \\
D^{\prime \prime \prime}=-X_{q} Q^{x}, \quad E^{\prime \prime}=0 ;
\end{array}
$$

et les équations de condition deviennent

$$
\begin{gathered}
X^{x} A=X^{x} X^{y} A=X^{y^{2}} A=X^{y^{2}} B=X^{y} X_{p} B=X_{p}^{2} B=X_{p} X_{q} B=0 \\
X_{p}^{2} C=X_{q}^{2} C=X^{x^{2}} D=X^{x} X_{q} D=X_{q}^{2} D=X_{p} X_{q} D=0 \\
X^{y} X_{p} A+X^{x} X^{y} B=2 X^{x} X_{q} A+X^{x^{y}} C=2 X^{y} X_{p} A+X^{y} C=0 \\
X^{x} X_{q} A+X^{x} X^{y} D=\left(X^{x} X_{p}-X^{y} X_{q}\right) B-X^{y} X_{p} C=X^{x} X_{q} C+ \\
+\left(X^{x} X_{p}-X^{y} X_{q}\right) D=0 \\
X_{p}^{2} A+2 X^{y} X_{p} C=X_{q}^{2} A+2 X^{x} X_{q} C=X_{q}^{2} B+X_{p} X_{q} C= \\
=X_{p} X_{q} C+X_{p}^{2} D=0 \\
X_{p} X_{q} A+2 X^{x} X_{q} B+\left(X^{x} X_{p}-X^{y} X_{q}\right) C=X_{p} X_{q} A+X^{x} X_{q} B+ \\
+X^{y} X_{p} D=0 \\
\left(X^{x} X_{p}-X^{y} X_{q}\right) A+X^{x^{2}} B-X^{x} X^{y} C=\left(X^{x} X_{p}-X^{y} X_{q}\right) A+ \\
+X^{x} X^{y} C-X^{y^{2}} D=0 .
\end{gathered}
$$

En écrivant

$$
\begin{aligned}
& \Delta_{x y} \equiv=X^{x} Y^{y}-X^{y} Y^{x}, \Delta_{x p} \equiv X^{x} Y_{p}-X_{p} Y^{x}, \Delta_{y p} \equiv X^{y} Y_{p}-X_{p} Y^{y} \text {, } \\
& \Delta_{p q} \equiv X_{p} Y_{q}-X_{q} Y_{p}, \quad \Delta_{x q} \equiv X^{x} Y_{q}-X_{q} Y^{x}, \quad \Delta_{y q} \equiv X^{y} \dot{Y}_{q}-X_{q} Y^{y} \text {, } \\
& \Delta_{i j}=-\Delta_{j i}
\end{aligned}
$$

on verifie sans difficulté que ces équations se réduisent aux équations sui- 
vantes :

$$
\begin{gathered}
X_{p} P^{x}-Y_{q} Q^{y}=0, \quad X_{q} P^{y}-Y_{p} Q^{x}=0, \quad \Delta_{y p}^{2}=0, \quad \Delta_{x q}^{2}=0 \\
Y_{p} Q^{x} \Delta_{q y}+Y_{q} Q^{y} \Delta_{x p}=0, \quad[X Y]^{2}+2\left|\Delta_{q x} \Delta_{y p}+2 \Delta_{p x} \Delta_{y q}\right|=0 \\
X_{q} Y_{p} \Delta_{q y}+X_{p} Y_{q} \Delta_{p x}=0, \quad X^{y} Y_{p} \Delta_{q y}+X_{p} Y^{y} \Delta_{p x}=0 \\
X^{x} Y_{p} \Delta_{p x}+X_{p} Y^{x} \Delta_{q y}+X^{x} Y_{q} \Delta_{y p}+X_{q} Y^{x} \Delta_{p y}+X^{y} Y_{q} \Delta_{x p}+ \\
+X_{q} Y^{y} \Delta_{y q}=0 \\
X^{x} Y_{q} \Delta_{y q}+X_{q} Y^{x} \Delta_{x p}=X y Y_{p} \Delta_{x p}+X_{p} Y^{y} \Delta_{y q}=X^{x} Y_{q} \Delta_{x p}+ \\
+X_{q} Y^{x} \Delta_{y q}=0 \\
X_{p} Y_{p} \Delta_{x p}+X_{q} Y_{p} \Delta_{y p}+X_{p} Y^{y} \Delta_{p q}=X_{q} Y_{q} \Delta_{q y}+X_{p} Y_{q} \Delta_{q x}+ \\
+X_{q} Y^{x} \Delta_{p q}=X^{y} Y^{x} \Delta_{q x}+X^{x} Y^{x} \Delta_{p x}+X^{x} Y_{q} \Delta_{x y}=0 ;
\end{gathered}
$$

ce système est équivalent au système suivant

$$
\begin{aligned}
& X_{p} P^{x}-Y_{q} Q^{y}=\Delta_{x y}=\Delta_{x p}=\Delta_{y p}=0, \\
& X_{q} P^{y}-Y_{p} Q^{x}=\Delta_{p q}=\Delta_{x q}=\Delta_{y q}=0 ;
\end{aligned}
$$

ce qui donne les transformations dualistiques.

En concluant on observe que le problème précédent et sa solution peuvent être generalisés pour un espace à $n+1$ dimensions.

En effet, soit

$$
X_{i}=X_{i}\left(x_{1}, x_{2}, \ldots, x_{n}, z\right), \quad Z=Z\left(x_{1}, x_{2}, \ldots, x_{n}, z\right), \quad i=1,2, \ldots, n,
$$

une transformation ponctuelle qui ne change pas l'équation

$$
\mathbf{\Sigma}_{i}^{n} \mathbf{\Sigma}_{j} p_{i, j} d x_{i} d x_{j}=0, \quad p_{i, j} \equiv \frac{\partial^{2} z}{\partial x_{i} \partial x_{j}}=\frac{\partial p_{i}}{\partial x_{j}}
$$

en vertu des identités

$$
d z=\sum_{1}^{n} p_{i} d x_{i}, \quad d p_{i}=\sum_{1}^{n} p_{i j} d x_{j}
$$

on a

$$
P_{i}=P_{i}\left(x_{1}, x_{2}, \ldots, x_{n}, z, p_{1}, p_{2}, \ldots, p_{n}\right)=\frac{\Delta^{(i)}}{\Delta}
$$


où

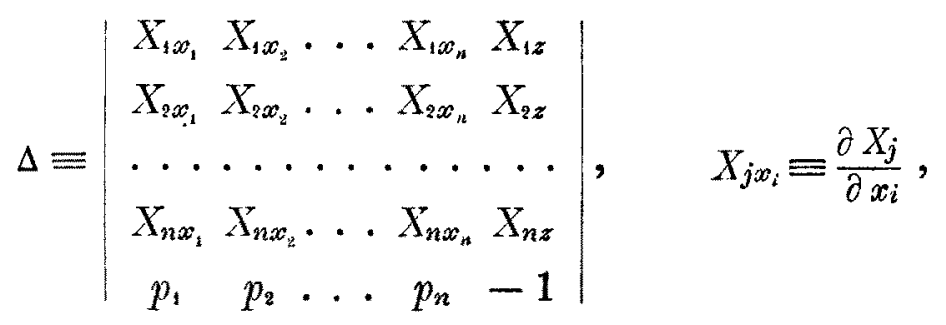

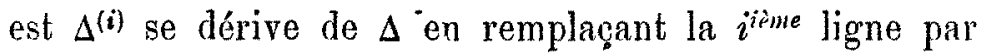

et aussi on trouve

$$
Z_{x_{1}} Z_{x_{2}} \ldots Z_{x_{11}} Z_{z}
$$

$$
\begin{gathered}
P_{i j}=P_{i j}\left(x_{1}, x_{2}, \ldots, x_{n}, z, p_{i}, p_{2}, \ldots, p_{n}, p_{41}, p_{i 2}, \ldots, p_{n n}\right)= \\
=\frac{D^{\prime i}}{\Delta}=\frac{D^{j}}{\Delta},
\end{gathered}
$$

où $D^{(i)}$ se forme de $\Delta$ quand on remplace la $i^{i \text { itme }}$ ligne par

D'ailleurs

$$
P_{i x_{1}} P_{i x_{2}} \ldots P_{i x_{n}} P_{i z} \text {. }
$$

$$
d X_{i}=X_{i z} d z+\sum_{1}^{n} X_{i x_{j}} d x_{j}
$$

et en construisant l'équation aux dérivées totales

$$
\mathbf{\Sigma}_{i}^{n} \mathbf{\Sigma}_{j} P_{i j} d X_{i} d X_{j}=0
$$

on roit qu'elle réduit à l'équation

$$
\begin{aligned}
& \Omega \equiv\left|\begin{array}{cccc}
X_{s x_{1}} & X_{1 x_{2}} & \ldots & X_{1 x_{n}} \\
X_{2 x_{1}} & X_{2 x_{2}} & \ldots & X_{2 x_{11}} \\
\ldots & \ldots & \cdots & . \\
X_{n x_{1}} & X_{n x_{2}} & \ldots & X_{n x_{n}}
\end{array}\right|^{2}
\end{aligned}
$$

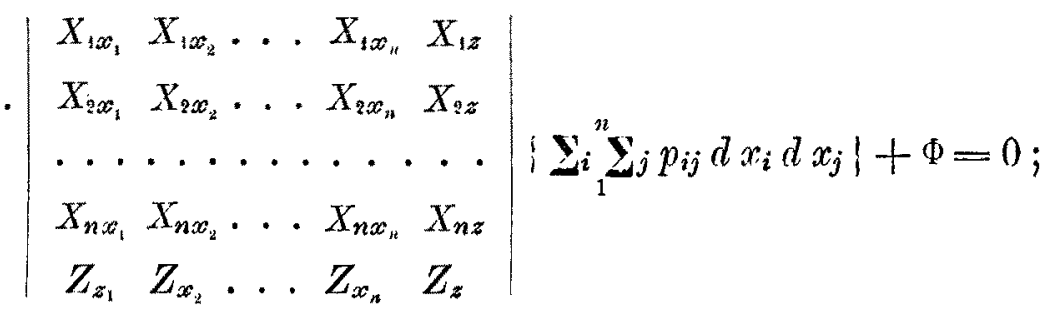


l'évanouissement identique pour toutes les valeurs de $p_{i}, p_{i j}$, de l'expansion de $\Omega$ donne le système suivant des équations aux dérivées partielles pour les fonctions inconnues:

$$
\begin{aligned}
& Z_{x_{i}} X_{j x_{i} x_{i}}-X_{j x_{i}} Z_{x_{i} x_{i}}=0
\end{aligned}
$$

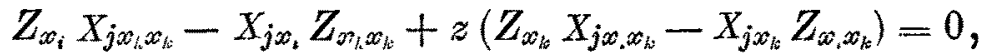

$$
\begin{aligned}
& Z_{x_{i}} X_{j x_{l} x_{l}}+Z_{x_{k}} X_{j x_{i} x_{l}}+Z_{x_{l}} X_{j x_{i} x_{k}}-X_{j x_{i}} Z_{x_{k_{k} x_{l}}-X_{j x_{k}}} Z_{x_{i} x_{l}}-X_{j x_{l}} Z_{x_{i} x_{k}}=0, \\
& x_{n+1}=z, \quad i, j, k, l=1,2, \ldots, n+1 \text {. }
\end{aligned}
$$

Il est commode pour l'intégration de ce système à diminuer le nombre des variables par unité et à écrire le système:

$$
\begin{gathered}
\varphi_{1 x_{i}} \varphi_{j x_{i} x_{i}}-\varphi_{j x_{i}} \varphi_{1 x_{i} x_{i}}=0, \\
\varphi_{1 x_{i}} \varphi_{j x_{k} x_{k}}-\varphi_{j x_{i}} \varphi_{1} x_{k} x_{k}+2\left(\varphi_{1} x_{k} \varphi_{j} x_{x_{i} x_{b}}-\varphi_{j x_{k}} \varphi_{1 x_{i} x_{k}}\right)=0,
\end{gathered}
$$

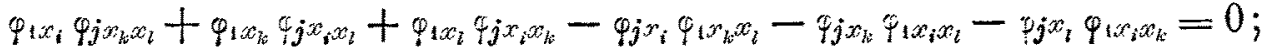

on déduit des équations (a) que

done

$$
\frac{\partial \log \varphi_{1 x_{j}}}{\partial x_{j}}=\frac{\partial \log \varphi_{2 x_{j}}}{\partial x_{j}}=\cdots=\frac{\partial \log \varphi_{n x_{j}}}{\partial x_{j}}, j=1,2, \ldots, n ;
$$

$$
\varphi_{i x_{j}}=\Phi^{i j}\left(x_{1}, x_{2}, \ldots, x_{j-1}, x_{j+1}, \ldots, x_{n}\right) \varphi_{1 x_{j}}, \quad i, j=1,2, \ldots, n .
$$

En substituant ces valeurs dans les équations $(b)$ on a

$$
\begin{gathered}
\left(\Phi^{i 1}-\Phi^{i j}\right) \varphi_{1 x_{1} x_{1}} \varphi_{i x_{j}}+2 \Phi_{x_{j}}^{i l}\left(\varphi_{i x_{1}}\right)^{2}=0, \\
\left(\Phi^{i j}-\Phi^{i i}\right) \varphi_{1 x_{j} x_{j}} \Phi_{i x_{1}}+2 \Phi_{x_{1}}^{i j}\left(\varphi_{1 x_{j}}\right)^{2}=0, \\
i, j=1,2, \ldots, n .
\end{gathered}
$$

Les équations (d) donnent

$$
\left(\Phi^{i j}-\Phi^{i k}\right) \varphi_{1 x_{j} x_{k}}+\Phi_{x_{k}}^{i j} \varphi_{1 x_{j}}-\Phi_{x_{j}}^{i k} \varphi_{1 x_{k}}=0, \quad i, j, k=1,2, \ldots, n,
$$

en vertu d'égalité

$$
\varphi_{i x_{j} x_{i}}=\varphi_{i x_{k} x_{j}} .
$$

En différentiant les équations $(f)$ par rapport à $x_{1}$ et $x_{j}$ on a

$$
\begin{aligned}
& \Phi_{x_{1}}^{i j} \varphi_{1 x_{1} x_{1}} \varphi_{1 x_{j}}-\left(\Phi^{i 1}-\Phi^{i j}\right)\left(\varphi_{1 x_{1} x_{1} x_{1} x_{1}}{ }_{1} x_{x_{j}}+\varphi_{1 x_{1} x_{1} \varphi_{1}} \varphi_{1 x_{i} x_{j}}\right)- \\
& -4 \Phi_{x_{j}}^{i l} \varphi_{1 x_{1}} \varphi_{1 x_{i} x_{1}}=0 \\
& \Phi_{x_{j}}^{i 1} \varphi_{1, x_{j} x_{j}} \varphi_{1 x_{1}}+\left(\Phi^{i 1}-\Phi^{i j}\right)\left(\varphi_{1 x_{j} x_{j} x_{j}} \varphi_{1 x_{2}}+q_{1} x_{x_{j} x_{j}} \varphi_{1 x_{2} x_{j}}\right)- \\
& -4 \Phi^{i j} \varphi_{i x_{j}} \varphi_{\varphi_{x j} x_{j}}=0 .
\end{aligned}
$$


En éliminant les quantités $\varphi_{1 x_{j} x_{j}}$ et $\varphi_{1 x_{j} x_{k}}$ des équations $(h)$ au moyen des équations $(f)$ et $(g)$ on trouve après une réduction facile

$$
\varphi_{i x_{j} x_{j} x_{j}}=\frac{6\left(\varphi_{i x_{j}}\right)^{3}\left(\Phi_{x_{k}}^{i j}\right)^{2}}{\left(\varphi_{i x_{k}}\right)^{2}\left(\Phi^{i j}-\Phi j^{j}\right)^{2}} .
$$

L'élimination des quantités

$$
\left(\Phi_{x_{i}}^{i j}\right)^{2} /\left(\Phi^{i j}-\Phi^{j k}\right)^{2}
$$

au moyen des équations (e) donne

$$
\frac{\varphi_{i x_{j} x_{j} x_{j}}}{\varphi_{i} x_{j} x_{j}}=\frac{3}{2} \frac{\varphi_{i x_{j} x_{j}}}{\varphi_{i} x_{j}}, \quad i, j=1,2, \ldots, n \text {; }
$$

en intégrant on trouve

$$
\begin{gathered}
\frac{\varphi_{i x_{j} x_{j}}}{\left(\varphi_{i x_{j}}\right)^{\frac{3}{2}}}=f_{i j}\left(x_{1}, x_{2}, \ldots, x_{j-1}, x_{j+1}, \ldots, x_{n}\right) ; \\
\varphi_{i x_{j}}=\frac{1}{\alpha_{i j}\left(x_{1}, x_{2}, \ldots, x_{j-1}, x_{j+1}, \ldots, x_{n}\right) x_{j}+\beta_{i j}\left(x_{1}, x_{2}, \ldots, x_{j-1}, x_{j+1}, \ldots, x_{n}\right)} ; \\
\text { et enfin } \\
\varphi_{i}=\frac{\gamma_{i j} x_{j}+\delta_{i j}}{\alpha_{i j} x_{j}+\beta_{i j}}, i, j=1,2, \ldots, n,
\end{gathered}
$$

où les formes

sont des fonctions de

$$
\alpha_{i j}, \beta_{i j}, \gamma_{i j}, \delta_{i j}
$$

$$
x_{i}, x_{2}, \ldots, x_{j-1}, x_{j+1}, \ldots, x_{n}
$$

done la fonction $\varphi_{i}$ est une fraction dont le numérateur et le dénominateur sont des fonctions linéaires des variables $x_{1}, x_{2}, \ldots, x_{n}$.

Les équations $(e)$ demandent que les dénominateurs soient les mêmes fonctions à une facteur prés; on le voit en supposant que

$$
\varphi_{i}=\frac{\alpha\left(x_{1}, x_{2}, \ldots, x_{n}\right)}{\gamma\left(x_{1}, x_{2}, \ldots, x_{n}\right)}, \quad \varphi_{j}=\frac{\beta\left(x_{1}, x_{2}, \ldots, x_{n}\right)}{\delta\left(x_{1}, x_{2}, \ldots, x_{n}\right)},
$$

où $\alpha, \beta, \gamma, \delta$ sont des fonctions linéaires par rapport aux $x_{1}, x_{2}, \ldots, x_{n} ;$ les équations (e) donnent

$$
\begin{gathered}
\frac{\varphi_{i x_{k}}}{\varphi_{j x_{k}}}=\frac{\gamma^{2}\left(\delta \beta_{x_{k}}-\beta \delta_{x_{k}}\right)}{\delta^{2}\left(\gamma \alpha_{x_{k}}-\alpha \gamma x_{k}\right)}=\frac{\Phi^{i 1}\left(x_{1}, x_{2}, \ldots, x_{k-1}, x_{k+1}, \ldots, x_{n}\right)}{\Phi^{j 1}\left(x_{1}, x_{2}, \ldots, x_{k-1}, x_{k+1}, \ldots, x_{n}\right)}= \\
\quad=\rho_{i j}\left(x_{1}, x_{2}, \ldots, x_{k-1}, x_{k+1}, \ldots, x_{n}\right), k=1,2, \ldots, n
\end{gathered}
$$

Annali di Matematica, Serie III, tomo VII. 
mais

$$
\frac{\delta \beta_{x_{k}}-\beta \delta_{x_{k}}}{\gamma \alpha_{x_{k}}-\alpha \gamma_{x_{k}}}=p_{k}\left(x_{1}, x_{2}, \ldots, x_{k-1}, x_{k+1}, \ldots, x_{n}\right)
$$

parce que les fonctions $\alpha, \beta, \gamma$, ठ sont linéaires par rapport à chacune variable; donc le rapport $\gamma: \delta$ est une constante.

Enfin les équations $(b)$ et $(c)$ interdisent que les produits $x_{k} x_{l}$ apparaissent dans $\varphi_{j}$; done on a

$$
\varphi_{i}=\frac{\sum_{j}^{n} \alpha_{i j} x_{j}+\alpha_{i n+1}}{\sum_{1}^{n} \beta_{j} x_{j}+\beta_{n+1}}
$$

Pour que déterminer les autres transformations de contact il ne faut considérer que les déterminants

où

$$
\begin{gathered}
\left|X_{1}^{x_{1}}, X_{2}^{x_{2}}, \ldots, X_{n}^{x_{n}}\right|, \quad\left|X_{1 p_{1}}, X_{2 p_{2}}, \ldots, X_{n p_{n}}\right| \\
\left|P_{1 p_{1}}, P_{2 p_{2}}, \ldots, P_{n p_{n}}\right|
\end{gathered}
$$

$$
K_{i}^{x_{j}}=K_{i x_{j}}+p_{j} K_{i z}, \quad K_{i x_{j}}=\frac{\partial K_{i}}{\partial x_{j}}, \quad K_{i p_{j}}=\frac{\partial K_{i}}{\partial p_{j}}
$$

les deux cas possibles donnent ou

ou

$$
X_{i p_{1}}=X_{i p_{2}}=\cdots=X_{i p_{n}}=X_{2 p_{1}}=\cdots=X_{n p_{n}}=0,
$$

$$
P_{t p_{1}}=P_{t p_{2}}=\cdots=P_{t p_{n}}=P_{2 p_{1}}=\cdots=P_{n p_{n}}=0
$$

c'est-à-dire les transformations projectives et les transformations dualistiques. 